\title{
Two-Degree-of-Freedom VIV of Circular Cylinder with Variable Natural Frequency Ratio: Experimental and Numerical Investigations
}

\author{
Narakorn Srinil, Hossein Zanganeh, Alexander Day \\ Department of Naval Architecture and Marine Engineering, University of Strathclyde, \\ Henry Dyer Building, Glasgow G4 0LZ, Scotland, UK
}

\section{Research Highlights}

- Two-degree-of-freedom VIV of circular cylinder with variable natural frequency ratio is experimentally investigated

- Experimental and numerical prediction results of coupled cross-flow and in-line responses are compared

- Good qualitative comparisons between experimental and numerical results are encouraged

- Experimental comparisons with other studies show good correspondence in amplitude and frequency responses

- Fundamental dual resonances of in-line/cross-flow responses are evidenced for all natural frequency ratios 


\title{
Two-Degree-of-Freedom VIV of Circular Cylinder with Variable Natural Frequency Ratio: Experimental and Numerical Investigations
}

\author{
Narakorn Srinil*, Hossein Zanganeh, Alexander Day \\ Department of Naval Architecture and Marine Engineering, University of Strathclyde, \\ Henry Dyer Building, Glasgow G4 0LZ, Scotland, UK
}

\begin{abstract}
Slender offshore structures possess multiple natural frequencies in different directions which can lead to different resonance conditions when undergoing vortex-induced vibration (VIV). This paper presents an experimental and numerical investigation of a two-degree-of-freedom VIV of a flexibly mounted circular cylinder with variable in-line-to-cross-flow natural frequency ratio. A mechanical spring-cylinder system, achieving a low equivalent mass ratio in both in-line and cross-flow directions, is tested in a water towing tank and subject to a uniform steady flow in a sub-critical Reynolds number range of about $2 \times 10^{3}-5 \times 10^{4}$. A generalized numerical prediction model is based on the calibrated Duffing-van der Pol (structure-wake) oscillators which can capture the structural geometrical coupling and fluid-structure interaction effects through system cubic and quadratic nonlinearities. Experimental results for six measurement datasets are compared with numerical results in terms of response amplitudes, lock-in ranges, oscillation frequencies, time-varying trajectories and phase differences of cross-flow/in-line motions. Some good qualitative agreements are found which encourage the use of the implemented numerical model subject to calibration and the sensitivity analysis of empirical coefficients. Moreover, comparisons of the newly-obtained and published experimental results are carried out and discussed, highlighting a good correspondence in both amplitude and frequency responses. Various patterns of figure-of-eight orbital motions associated with dual two-to-one resonances are observed experimentally as well as numerically: the forms of trajectories are noticed to depend on the system mass ratio, damping ratio, reduced velocity parameter and natural frequency ratio of the two-dimensional oscillating cylinder.
\end{abstract}

Keywords: Vortex-induced vibration, Cross-flow/in-line motion, Circular cylinder, Experimental investigation, Numerical prediction

* Corresponding author: narakorn.srinil@strath.ac.uk ; Tel. +44 1415483463; Fax +44 1415522879 


\section{Nomenclature}

$\begin{array}{ll}A_{x} / D, A_{y} / D & \text { Dimensionless in-line and cross-flow amplitudes } \\ A_{x m} / D, A_{y m} / D & \text { Dimensionless maximum attainable amplitudes } \\ C_{D}, C_{L} & \text { Fluctuating drag and lift coefficients of an oscillating cylinder } \\ C_{D 0}, C_{L 0} & \text { Fluctuating drag and lift coefficients of a stationary cylinder } \\ C_{M} & \text { Potential added mass coefficient } \\ D & \text { Cylinder diameter } \\ f^{*} & \text { Cylinder in-line to cross-flow natural frequency ratio } \\ F_{D}, F_{L} & \text { Fluctuating drag and lift forces } \\ F_{x}, F_{y} & \text { Hydrodynamic forces in streamwise and transverse directions } \\ f_{n x}, f_{n y} & \text { Natural frequency in still water of cylinder } \\ f_{o x}, f_{o y} & \text { Dominant frequency of the oscillating cylinder } \\ L_{c}\left(L_{p}\right) & \text { Cylinder submerged (pendulum) length } \\ L_{c} / D & \text { Aspect ratio } \\ M_{D}, M_{L} & \text { System mass parameters } \\ m_{f} & \text { Fluid added mass } \\ m_{s} & \text { Cylinder mass } \\ p, q & \text { Reduced vortex drag and lift variables } \\ \mathrm{Re}_{\mathrm{S}} & \text { Reynolds number } \\ \mathrm{S}_{\mathrm{GX}}, \mathrm{S}_{\mathrm{GY}} & \text { Strouhal number } \\ t & \text { Skop-Griffin mass-damping parameter } \\ T & \text { Dimensionless time } \\ V & \text { Dimensional time } \\ V_{r} & \text { Uniform flow velocity } \\ \tilde{Y} & \text { Reduced velocity parameter } \\ X, Y & \text { Dimensional transverse displacement } \\ x, y & \text { Streamwise and transverse coordinates } \\ \alpha_{x}, \alpha_{y}, \beta_{x}, \beta_{y} & \text { Dimensionless in-line and cross-flow displacements } \\ \gamma & \text { Dimensionless geometrically-nonlinear coefficients } \\ \varepsilon_{x}, \varepsilon_{y} & \text { Stall parameter } \\ \theta & \text { Wake oscillator coefficients } \\ \theta_{x}, \theta_{y} & \text { Direction of effective lift (drag) force measured from } Y(X) \text { axis } \\ \lambda_{x}, \lambda_{y} & \text { Phase angles } \\ \Lambda_{x}, \Lambda_{y} & \text { Combined fluid-structural damping terms } \\ \mu, m^{*} & \text { Wake-cylinder coupling coefficients } \\ \xi_{x}, \xi_{y}, \xi & \text { Mass ratios } \\ \rho & \text { Structural reduced damping or damping ratios } \\ \Omega & \text { Fluid density } \\ & \end{array}$




\section{Introduction}

Vortex-induced vibration (VIV) has received a considerable amount of interest over the years due to the variety of nonlinear phenomena governed by the fluid mechanics, structural dynamics and fluid-structure interactions. In many ocean and offshore engineering applications, VIV continues to be of great concern in the context of fatigue analysis, design and operation of deep water structures exposed to ocean currents. From a theoretical and practical viewpoint, both experimental tests and numerical prediction models - capable of capturing VIV occurrences and behaviors in a wide range of both the hydrodynamics and the structural parameters - are important. However, in spite of many published studies, the vast majority of the research literature has focused on one-dimensional cross-flow VIV of a circular cylinder for which the transverse response is typically observed to be the largest ADDIN EN.CITE (, and on the related semi-empirical modelling of a 1-degree-of-freedom (DOF) cross-flow-only VIV (Gabbai and Benaroya, 2005). While there are some recent computational fluid dynamics and flow visualization studies to advance the comprehension of VIV phenomena (Bao et al., 2012; Jauvtis and Williamson, 2003; Jeon and Gharib, 2001; Williamson and Jauvtis, 2004), experimental investigations and comparisons with numerical prediction results for two-dimensional in-line $(X)$ and cross-flow $(Y)$ or 2-DOF VIV are still rather limited (Hansen et al., 2002; Pesce and Fujarra, 2005; Stappenbelt and O'Neill, 2007) and therefore needed to be further addressed comprehensively.

In this study, new experimental VIV results of a 2-DOF circular cylinder with equivalent mass ratio in both $X-Y$ directions $\left(m_{x}^{*}=m_{y}^{*}\right)$ and variable in-line-to-cross-flow natural frequency ratio $\left(f^{*}=f_{n x} / f_{n y}\right)$ are presented and compared with the associated numerical outcomes predicted by new nonlinear structure-wake oscillators (Srinil and Zanganeh, 2012). Some insightful VIV aspects are also discussed in the light of other published experimental results with variable $f^{*}$ but $m_{x}^{*} \neq m_{y}^{*}$ (Dahl et al., 2006). Note that the condition of $m_{x}^{*}=m_{y}^{*}$ is more relevant in practice than that of $m_{x}^{*} \neq m_{y}^{*}$ to real cylindrical offshore structures including risers, mooring cables and pipelines. The $f^{*}$ variation is also of practical relevance because such a distributed-parameter system contains an infinite number of natural frequencies in different directions entailing various $f^{*}$ (Srinil and Rega, 2007; Srinil et al., 2007). These can result in different lock-in or resonant conditions with the vortex shedding frequencies of the fluctuating lift and drag forces. As the drag oscillation has double the frequency of the lift oscillation, a perfect two-dimensional resonance case might occur 
when $f^{*}=2$. This circumstance could lead to a large-amplitude response for a system with low mass and damping.

Recent experimental studies have highlighted some interesting features of 2-DOF VIV of circular cylinders and meaningful contributions from the in-line VIV to the overall dynamics, depending on several control parameters. In general, the freedom of the system to oscillate in the in-line direction can cause an increase of the cross-flow response amplitude and widen the lockin range (Moe and Wu, 1990; Sarpkaya, 1995); it has been suggested that these effects may result from an enhanced correlation of the transverse force along the cylinder span (Moe and $\mathrm{Wu}$, 1990). With respect to the flow field visualization, a new 2 T (two of vortex triplets) wake mode has been observed for the cylinder with significant combined $X-Y$ motion (Williamson and Jauvtis, 2004) in addition to the typical 2S (two single vortices) and 2P (two vortex pairs) modes defined in the $Y$-only cylinder motion case (Khalak and Williamson, 1999). In the framework of 2-DOF forced vibration where the amplitudes and oscillation frequencies $\left(f_{o x}\right.$ and $\left.f_{\text {oy }}\right)$ of the cylinder are specified a priori, Jeon and Gharib (2001) found that, in the case of $f_{o x} / f_{\text {oy }}=2$, a small amount of in-line motion can inhibit the vortex formation of the $2 \mathrm{P}$ mode. They also suggested a possible change in the relative phases between the lift and drag forces: this implies the possible energy transfer between the oscillating body and the wake forces in the free vibration case. Recently, Laneville (2006) proposed that the level of $X-Y$ motion depends on a ratio of the time derivatives between in-line and cross-flow responses.

With $m_{x}^{*}=m_{y}^{*}$ and $f^{*}=1$, Jauvtis and Williamson (2004) showed that there is a slight influence on the cross-flow response of the cylinder with $m^{*}>6$ when comparing the results obtained between 1- and 2-DOF models. When $m^{*}<6$, there is a super-upper branch in the cross-flow response with the peak amplitude $A_{y} / D \approx 1.5$ coexisting with the in-line response with the peak amplitude $A_{x} / D \approx 0.3$, along with response jump and hysteresis phenomena. Similar nonlinear responses and ranges of maximum $A_{x} / D$ and $A_{y} / D$ have been experimentally reported by Stappenbel et al. (2007) and Belvins and Coughran (2009), and numerically captured by Zhao et al. (2011). A two-dimensional lock-in range is found to be mainly influenced by the variation of the mass ratio (Stappenbelt et al., 2007). However, both mass (Stappenbelt et al., 2007) and damping (Blevins and Coughran, 2009) parameters can influence on 2-DOF peak amplitudes as in the 1-DOF cases (Khalak and Williamson, 1999).

With $m_{x}^{*} \neq m_{y}^{*}$ and $f^{*} \neq 1$, different qualitative and quantitative features of 2-DOF VIV responses appear. In particular, a two-peak cross-flow response has been noticed by Sarpkaya (1995) and 
Dahl et al. (2006) with $f^{*=}=2$ and 1.9, respectively. Dahl et al. (2010) further highlighted various figure-of-eight patterns in different subcritical and supercritical Reynolds number (Re) ranges $\left(1.5 \times 10^{4}<\operatorname{Re}<6 \times 10^{4}\right.$ and $\left.3.2 \times 10^{5}<\operatorname{Re}<7.1 \times 10^{5}\right)$ and described a figure-eight occurrence as a representation of "dual resonance”. Under this dual resonance, the frequencies of the unsteady drag and lift forces are resonantly tuned with $f_{o x}$ and $f_{o y}$, respectively, such that $f_{o x} / f_{o y} \approx 2$. In addition, a large third harmonic component of the lift force was observed although the main cross-flow response was primarily associated with the first-harmonic lift force. This is in agreement with a direct numerical simulation work by Lucor and Triantafyllou (2008) and a lab experiment of a flexible cylinder by Trim et al. (2005).

In spite of the above-mentioned studies, the most practical case of $m_{x}^{*}=m_{y}^{*}$ and variable $f^{*}$ has not been thoroughly investigated. For a particular flow with very low $\operatorname{Re}=150$ and zero structural damping, Bao et al. (2012) recently have performed direct numerical simulations of a circular cylinder with $f^{*}=1,1.25,1.5,1.75$ and 2 . They showed dual resonances in all $f^{*}$ cases and illustrated how the oscillating drag component is maximized when $f^{*}=2$ with the appearance of the $\mathrm{P}+\mathrm{S}$ vortex wake mode associated with the maximum in-line response. Nevertheless, more experimental and numerical investigations in a higher Re range are still needed along with the improvement of relevant prediction models.

The main objectives of the present study are to (i) experimentally investigate 2-DOF VIV of a flexibly mounted circular cylinder with $m_{x}^{*}=m_{y}^{*}$ and variable $f^{*}$; (ii) compare the obtained experimental results with numerical prediction outcomes in order to improve the newly-proposed coupled oscillators (Srinil and Zanganeh, 2012) with a proper choice of system coefficients; and (iii) compare various observations from the present experimental campaign with other studies. To calibrate model empirical coefficients, particular attention is placed on the determination of cylinder maximum attainable amplitudes, associated lock-in ranges (both the onset and the end of synchronization), two-dimensional orbital $X$ - $Y$ motions and oscillation frequencies, by comparing various cases of $f^{*} \neq 1$ and $f^{*}=1$. These analysis outcomes based on a 2-DOF rigid cylinder could be practically useful in the improvement of VIV prediction tools and design guidelines for crossflow/in-line VIV of flexible cylinders with multi DOF and various $f^{*}$, as conducted, for instance, by Srinil (2010, 2011) for cross-flow-only VIV cases.

This paper is structured as follows. In Section 2, details of the new experimental arrangement used for the 2-DOF VIV study of a flexibly mounted circular cylinder are presented along with the test matrix. The associated numerical prediction model and governing equations are explained 
in Section 3. Depending on system parameters, comparisons of experimental and numerical prediction results of six measurement datasets are made in Section 4 which also demonstrates the sensitivity analysis and the influence of key geometrical parameters on VIV predictions. In Section 5, various new and published experimental results are compared and discussed. Some insightful aspects from a mathematical modelling, numerical prediction and experimental viewpoint are summarized in Section 6. The paper ends with the conclusions in Section 7.

\section{Experimental Arrangement and Test Matrix}

A new experimental test rig for the study of 2-DOF VIV of a flexibly mounted, smooth and rigid circular cylinder subject to a uniform steady flow has been developed for use in the towing tank at the Kelvin Hydrodynamics Laboratory (KHL) of the University of Strathclyde, Glasgow, UK. The design of this rig was motivated by a recent collaborative work conducted at the University of Sao Paulo, Brazil (Assi et al., 2012). The KHL tank has dimensions of $76 \mathrm{~m}$ long by $4.57 \mathrm{~m}$ wide; water depth can be varied from $0.5-2.3 \mathrm{~m}$. The tank is equipped with a self-propelled towing carriage on which the experimental apparatus can be firmly installed, and a variety of damping systems to calm the water rapidly between runs.

Figure 1 displays the experimental set-up where the test cylinder is mounted vertically and connected at its upper end to a long aluminum pendulum with total length of about $4.1 \mathrm{~m}\left(L_{p}\right)$. The pendulum is attached to the supporting framework via a high-precision universal joint at the top of the frames. The test cylinder adopted in the present study is made of thick-walled cast nylon tube, having an outer diameter $(D)$ of $114 \mathrm{~mm}$ and a fully submerged length $\left(L_{c}\right)$ of 1.037 $\mathrm{m}$. The lower end of the cylinder is located $50 \mathrm{~mm}$ from the bottom of the tank, and the upper end is located $50 \mathrm{~mm}$ beneath the static free surface. Such lower end condition was deemed to produce a negligible effect on the peak amplitudes (Morse et al., 2008). It should be noted that the pendulum effect on the uniformity of the local flow field is believed to be insignificant since the maximum roll and pitch angles of the cylinder about the universal joint were found to be only about 2 degrees in all tests. The blockage is about $2.5 \%$ and the aspect ratio $\left(L_{c} / D\right)$ of the cylinder is about 9 being comparable to $L_{c} / D$ in some recent studies (Jauvtis and Williamson, 2004; Sanchis et al., 2008; Stappenbelt et al., 2007).

The mechanical system is restrained to allow the cylinder to oscillate freely with arbitrary amplitudes in both in-line $(X)$ and cross-flow $(Y)$ directions by using two pairs of coil springs (with lengths of about $50 \mathrm{~cm}$ ) rearranged perpendicularly in the horizontal $X$ - $Y$ plane. Each spring obeys Hooke’s law (i.e. with a linear constant stiffness); nevertheless, as the cylinder oscillates 
two-directionally due to VIV, the assembly creates the geometrically nonlinear coupling of crossflow/in-line displacements. These non-linear effects are accounted for in the numerical prediction model (see Section 3). Measurement of cylinder motions was carried out using a Qualisys optical motion capture system with a fixed sampling frequency of $137 \mathrm{~Hz}$. Four infrared cameras were used to identify and optimize the three-dimensional positions of several reflective markers mounted on the pendulum, and calibration was performed with an average residual across all cameras of less than $0.3 \mathrm{~mm}$. Key outputs were the roll and pitch angles with a degree resolution of 0.001. In contrast with traditional displacement measurement instruments, the non-contact nature of this system ensures that no unwanted additional damping or restoring forces are applied to the pendulum. Note that establishing the systematic uncertainty of measurements of the optical motion-tracking systems is more challenging than the contact-based measurements since this uncertainty depends upon the position and orientation of both the cameras and the reflective markers. Procedures for formal assessment of uncertainty for use in future campaigns are currently being developed. The acquisition time for each steady-state response was about 2 minutes and the waiting time between each two consecutive measurements was about 5 minutes. A trailing wheel of very accurately defined circumference was attached to the carriage, and the angular velocity of the wheel was determined using a high-precision magnetic encoder and a counter-time which outputs the velocity signal representing the carriage speed.

When the cylinder is towed, the mean drag causes a mean in-line displacement of the cylinder in the flow direction; however, only the fluctuating displacements are of main interest. In order that the mean position of the cylinder for the measurements is vertical in the in-line direction to avoid the possible cylinder inclination effect on VIV, this displacement was initially adjusted by pre-tensioning the upstream in-line spring such that the cylinder mean position, as measured using the Qualisys system, remains nearly vertical during the VIV test. While the pre-tension is meaningful in the evaluation of hydrodynamic forces which is beyond the scope of this study, overall VIV measurements were conducted after such an adjusted equilibrium position was achieved. A thorough treatment of a similar spring arrangement is contained in Stappenbelt (2010).

As actual slender structures have multiple natural frequencies in different directions (Srinil et al., 2007), attention in the present study is placed on the cylinder model with varying ratios between in-line $\left(f_{n x}\right)$ and cross-flow $\left(f_{n y}\right)$ natural frequencies in still water $\left(f^{*}=f_{n x} / f_{n y}\right)$. This was achieved in practice by using springs with differing stiffness. The reported experimental crossflow $\left(A_{y} / D\right)$ and in-line $\left(A_{x} / D\right)$ amplitudes normalized by the cylinder diameter are referred to as the maximum displacements at the bottom tip of the cylinder. Based on a free decay test in air, 
the experimental apparatus with and without the cylinder-spring system was found to be lightly damped at around $0.5 \%$ and $0.2 \%$ of the critical damping, respectively. A series of free decay tests in calm water were performed to identify $f_{n x}, f_{n y}$ and the associated damping ratios $\left(\xi_{x}, \xi_{y}\right)$ in both $X$ and $Y$ directions. Small initial displacements were assigned independently in each $X$ or $Y$ direction to ensure that no geometric nonlinear coupling took place: $f_{n x}$ and $f_{n y}$ were obtained from the free damped responses whose maximum amplitudes were about 0.1 of the diameter in all datasets. The representative averaged $\xi_{x}$ and $\xi_{y}$ values have been evaluated by subtracting the fluid damping component from the total damping of the system (Sumer and Fredsoe, 2006).

Table 1 summarizes a test matrix of 6 datasets (labeled as KHL1-KHL6) in which two mass ratios $\left(m_{x}^{*}=m_{y}^{*}=m^{*}=1.4\right.$ and 3.5) of the cylinder are considered. These $m^{*}$ were considered to be low, being less than 6 (Jauvtis and Williamson, 2004), to encourage the effect of in-line VIV and the overall large-amplitude responses. Due to the amplitude-dependence nature of the structural and fluid-added damping in water, variable $\xi_{x}$ and $\xi_{y}$ values (between 1-5 \%) are reported. The combined mass-damping values are in the range of $0.014<m^{*} \xi<0.081$. The $m^{*}=1.4$ case (KHL1-KHL5) corresponds to the initial apparatus setup, whilst in later tests $m^{*}$ was increased by adding lump masses to the rig system such that $m^{*}=3.5$ (KHL6). Such an increased $m^{*}$ case allows us to evaluate the prediction model (Section 4) whose empirical coefficients have been calibrated based on the experiments with varying $m^{*}$ (Stappenbelt et al., 2007). For $m^{*}=1.4$, five tests with different $f^{*} \approx 1.0,1.3,1.6$ and 1.9 were performed to justify the occurrence of a dual 2:1 resonance regardless of $f^{*}$ and as the drag fluctuation has double the frequency of the lift fluctuation. In all datasets, the reduced velocity $V_{r}$ range in which $V_{r}=V / f_{n y} D$ was about $0<V_{r}<20$, corresponding to $2 \times 10^{3}<\operatorname{Re}<5 \times 10^{4}$ of the sub-critical flows and the flow speed $V$ of 0.02-0.6 $\mathrm{m} / \mathrm{s}$. This considered range encompassed a $V_{r}$ value at which the peak amplitude occurred. Some tests were repeated in the neighborhood of peaks and response jumps.

With the aim of comparing our experimental results with other published studies by also focusing on the variation of $f^{*}$, the experimental model performed at the MIT towing tank (Dahl et al., 2006) is herein considered. Their test matrix, comprising 6 datasets (labeled as MIT1MIT6) with $L_{c} / D$ of $26,0.041<m^{*} \xi<0.353$, and $11 \times 10^{3}<\operatorname{Re}<6 \times 10^{4}$, is given in Table 2 in comparison with KHL datasets in Table 1. It is worth noting that both experiments have similar $\xi_{x}$ and $\xi_{y}$ values in the range of about 1-6 \%. The role of damping will be again discussed in Section 6. Apart from being different in the experimental arrangement and procedure, in $L_{c} / D$, and variable $\xi$ and $f^{*}$ values, the main distinction between KHL and MIT datasets is due to the 
specified mass ratios: $m_{x}^{*}=m_{y}^{*}$ in this study whereas $m_{x}^{*} \neq m_{y}^{*}$ in Dahl et al. (2006). This aspect along with other observations will be taken into account in the comparison of results in Section 5.

\section{Numerical Model with Nonlinear Coupled Structure-Wake Oscillators}

The capability to reasonably model and predict the VIV structural response excited by the unsteady flow field has been a major challenge to modelers and engineers for many years. Recently, a new semi-empirical model for predicting 2-DOF VIV of a spring-mounted circular cylinder in a uniform steady flow has been developed and calibrated with some published experimental results in the cases of $m_{x}^{*}=m_{y}^{*}$ and $f^{*}=1$ (Srinil and Zanganeh, 2012). As an extended study, the same model is improved and utilized to predict cross-flow/in-line VIV responses in the cases of variable $f^{*}$, based on calibration with new in-house experimental results. Some modified empirical coefficients will be then suggested in Section 4 for a future use.

A schematic model of the cylinder restrained by two pairs of springs to oscillate in $X$ and $Y$ directions is displayed in Fig. 2a. The key aspect in the formulation of system equations of motions is to capture the quadratic relationship between in-line and cross-flow displacements (Vandiver and Jong, 1987). Following Wang et al. (2003), the two-directional unsteady fluid forces are exerted on the oscillating cylinder as opposed to the stationary one, by also accounting for the relative velocities between the incoming flow and the cylinder in-line motion. As a result, the instantaneous lift $\left(F_{L}\right)$ and drag $\left(F_{D}\right)$ forces coincide with an arbitrary plane making up an angle of $\theta$ with respect to the $Y$ and $X$ axes, respectively. Two cases can be realized depending on whether $\theta$ is counterclockwise (Fig. 2b) or clockwise (Fig. 2d). From our numerical simulation experience, it has been discovered that such $\theta$ direction plays a key role in the ensuing phase difference between cross-flow and in-line oscillations and, correspondingly, the figure-of-eight appearing shape. In general, the orbital plot exhibits a figure-eight trajectory with tips pointing upstream with a counterclockwise $\theta$ model (e.g. Fig. 2c) or downstream with a clockwise $\theta$ model (e.g. Fig. 2e). As both cases have been experimentally observed in the literature depending on the system parameters, they are herein accounted for in the improved model formulation. Note that only the counterclockwise $\theta$ model was proposed in Srinil and Zangeneh (2012).

Consequently, by assuming a small $\theta$, the unsteady hydrodynamic forces $F_{x}$ and $F_{y}$ may be simplified and expressed after resolving $F_{L}$ and $F_{D}$ into the $X$ and $Y$ directions as

$$
F_{x}=F_{D} \cos \theta \mp F_{L} \sin \theta \approx F_{D} \mp F_{L} \dot{\tilde{Y}} / V,
$$




$$
F_{y}=F_{L} \cos \theta \pm F_{D} \sin \theta \approx F_{L} \pm F_{D} \dot{\tilde{Y}} / V
$$

where $\tilde{Y}$ is the dimensional transverse displacement, a dot denotes differentiation with respect to the dimensional time $T, F_{D}=\rho D V^{2} C_{D} / 2, F_{L}=\rho D V^{2} C_{L} / 2, \rho$ is the fluid density, $C_{D}$ and $C_{L}$ are the time-varying drag and lift coefficients, the minus (positive) and positive (minus) sign in Eq. (1) (Eq. (2)) corresponds to the case of counterclockwise and clockwise $\theta$, respectively.

By assigning the fluid vortex variables as $p=2 C_{D} / C_{D 0}$ and $q=2 C_{L} / C_{L 0}$ (Facchinetti et al., 2004) in which $C_{D 0}$ and $C_{L 0}$ are the associated oscillating drag and lift coefficients of a stationary cylinder (assumed as $C_{D 0}=0.2$ (Currie and Turnbull, 1987) and $C_{L 0}=0.3$ (Blevins, 1990)), the time variation of $p$ and $q$ may be assumed to follow the self-excitation and -limiting mechanism of the van der Pol wake oscillator (Bishop and Hassan, 1964). By introducing the dimensionless time $t=\omega_{n y} T$ and normalizing the displacements with respect to $D$, the nonlinearly coupled equations describing the in-line $(x)$ and cross-flow $(y)$ oscillations of the cylinder subject to the fluctuating fluid force components $(p, q)$ are expressed in dimensionless forms as

$$
\begin{aligned}
& \ddot{x}+\lambda_{x} \dot{x}+f^{* 2}\left(x+\alpha_{x} x^{3}+\beta_{x} x y^{2}\right)=M_{D} \Omega^{2} p \mp 2 \pi M_{L} \Omega^{2}\left(q \dot{y} / V_{r}\right), \\
& \ddot{p}+2 \varepsilon_{x} \Omega\left(p^{2}-1\right) \dot{p}+4 \Omega^{2} p=\Lambda_{x} \ddot{x}, \\
& \ddot{y}+\lambda_{y} \dot{y}+y+\alpha_{y} y^{3}+\beta_{y} y x^{2}=M_{L} \Omega^{2} q \pm 2 \pi M_{D} \Omega^{2}\left(p \dot{y} / V_{r}\right), \\
& \ddot{q}+\varepsilon_{y} \Omega\left(q^{2}-1\right) \dot{q}+\Omega^{2} q=\Lambda_{y} \ddot{y},
\end{aligned}
$$

in which $M_{D}=C_{D 0} / 16 \pi^{2} \mathrm{St}^{2} \mu, \quad M_{L}=C_{L 0} / 16 \pi^{2} \mathrm{St}^{2} \mu, \quad \mu=\left(m_{s}+m_{f}\right) / \rho D^{2}, \quad \lambda_{x}=2 \xi_{x} f^{*}+\gamma \Omega / \mu$, $\lambda_{y}=2 \xi_{y}+\gamma \Omega / \mu, \Omega=\mathrm{St} V_{r}, m_{f}=\pi \rho D^{2} C_{M} / 4, m_{s}$ is the cylinder mass, $m_{f}$ the fluid added mass, $C_{M}$ the potential added mass coefficient assumed to be unity for a circular cylinder (Blevins, 1990), St the Strouhal number, $\gamma$ the stall parameter which is directly related to the sectional mean drag coefficient and assumed to be a constant equal to 0.8 (Facchinetti et al., 2004), and co-subscripts $x$ and $y$ identify properties in these directions. Note that the mass ratio definition in the literature is variable but the widely recognized one with $m^{*}=4 \mu / \pi-C_{M}$ is herein considered (Williamson and Govardhan, 2004).

In contrast to typical VIV models which consider a linear structural oscillator to describe the cylinder displacement (Gabbai and Benaroya, 2005), Eqs. (3) and (5) account for the effects of geometric and hydrodynamic nonlinearities on the oscillating cylinder. These equations are socalled Duffing oscillators (Nayfeh, 1993). It is also worth mentioning the following key points. 
i. Cubic nonlinear terms capture the effect of nonlinear stretching $\left(x^{3}, y^{3}\right)$ and physical coupling of cross-flow and in-line displacements $\left(x y^{2}, x^{2} y\right)$, depending on the geometrical parameters $\left(\alpha_{x}, \alpha_{y}, \beta_{x}, \beta_{y}\right)$.

ii. Quadratic nonlinear terms $(q \dot{y}, p \dot{y})$ capture the effect of wake-cylinder interaction; these have been found to be responsible for the figure-of-eight appearance associated with a dual 2:1 resonance (Srinil and Zanganeh, 2012).

iii. In Eqs. (3) and (5), the maximum cross-flow/in-line amplitudes are unaffected by the choice of $\theta$ since the associated velocities are trivial, making $q \dot{y} / V_{r} \approx p \dot{y} / V_{r} \approx 0$.

iv. In qualitative agreement with a 1-DOF VIV study of Facchinetti et al. (2004), the linear coupling terms based on the cylinder accelerations $\left(\Lambda_{x} \ddot{x}, \Lambda_{y} \ddot{y}\right)$ in Eqs. (4) and (6) have been found to produce a better 2-DOF VIV prediction than the displacement $\left(\Lambda_{x} x, \Lambda_{y} y\right)$ and velocity $\left(\Lambda_{x} \dot{x}, \Lambda_{y} \dot{y}\right)$ models (Zanganeh and Srinil, 2012).

v. The mean drag force component is omitted from Eq. (1) and (3) by assuming that it does not affect the fluctuating displacement components as considered by Kim and Perkins (2002). However, the nonlinear terms in Eq. (3) can generate the in-line static drift (Nayfeh, 1993) of the cylinder; this drift is disregarded from the numerical simulations as attention is placed on the evaluation of the oscillating amplitude components.

The analysis of coupled cross-flow/in-line VIV depends on several empirical coefficients $\left(\varepsilon_{x}\right.$, $\left.\varepsilon_{y}, \Lambda_{x}, \Lambda_{y}\right)$ and geometrical parameters $\left(\alpha_{x}, \alpha_{y}, \beta_{x}, \beta_{y}\right)$. In this study, $\alpha_{x}, \alpha_{y}, \beta_{x}$ and $\beta_{y}$ are also treated as empirical coefficients to account for the time- and amplitude-dependent uncertainties during the experiment such as the spatial correlation of vortices along the cylinder span, the fluidadded damping, the free surface effect and the wake-cylinder interaction. Based on calibration with experimental results (Stappenbelt et al., 2007) with $f^{*}=1$ and varying $m^{*}$, it may be assumed that (Srinil and Zanganeh, 2012, 2013) $\varepsilon_{y}=0.00234 e^{\left(0.228 m^{*}\right)}$.

To reduce the time-consuming task involving the tuning of individual coefficients, $\varepsilon_{x}=0.3, \Lambda_{x}$ $=\Lambda_{y}=12$, and $\alpha_{x}=\alpha_{y}=\beta_{x}=\beta_{y}=0.7$ are initially assumed in the $f^{*}=1$ cases following Srinil and Zanganeh (2012). However, some of these values will be modified in Section 4 based on the comparisons with experimental results in the $f^{*} \neq 1$ cases. Nonlinear coupled Eqs. (3)-(6) can be numerically solved by using a fourth-order Runge-Kutta scheme with an adaptive time step enabling solution convergence and stability, and with assigned initial conditions at $t=0$ of $x=y$ 
$=0, p=q=2$ and zero velocities. In all numerical simulation cases, $V_{r}$ is increasingly varied in steps of 0.1 and at least 60 cycles of the vortex shedding frequency are accounted for in the evaluation of steady-state responses.

\section{Comparisons of Experimental and Numerical Prediction Results}

Experimental and numerical prediction results are now compared based on KHL data in Table 1. As typical figure-of-eight orbital motions with tips pointing downstream (e.g. Fig. 2e) are mostly observed in the present experiments, the system equations of motions (Eqs. 3-6) used in numerical simulations are based on the model configuration shown in Fig. 2d. To facilitate the comparison and discussion, two sets of results are classified depending on $f^{*}$ : (i) $f^{*}=1$ (KHL1, KHL5, KHL6) and (ii) variable $f^{*}$ with $f^{*}=1.29$ (KHL2), 1.60 (KHL3) and 1.85 (KHL4). Both maximum and root-mean-squared (RMS) values of cross-flow $\left(A_{y} / D\right)$ and in-line $\left(A_{x} / D\right)$ amplitudes are evaluated.

Results in the $f^{*}=1$ cases are plotted in Fig. 3 which illustrates a fairly good qualitative comparison of numerical (lines) and experimental (symbols) responses. From the experiments, pure in-line responses are observed in a marginal range of about $2<V_{r}<4$ (Fig. 3b, d and f) whereas coexisting cross-flow/in-line VIV responses take place in the range of about $4<V_{r}<$ 17.5 (Fig. 3a and c) or $4<V_{r}<12.5$ (Fig. 3e), depending on $m^{*}$. As expected from both a numerical and experimental viewpoint, both KHL1 and KHL5 datasets with the lower $m^{*}=1.4$ exhibit a wider synchronization region. With increasing $V_{r}$, some jumps of peak amplitudes from upper to lower branches (Figs. 3c-f) are experimentally as well as numerically (denoted by vertical dashed lines) observed. These jumps are in agreement with several recently published experimental results of 2-DOF VIV with $f^{*}=1$ (Blevins and Coughran, 2009; Jauvtis and Williamson, 2004; Stappenbelt et al., 2007).

In view of quantitative comparisons, the highest values of experimental and numerical RMS amplitudes are found to be comparable in the range of about 0.9-1.25 for $A_{y} / D$ (Fig. 3a, c and e) and 0.1-0.3 for $A_{x} / D$ (Fig. 3b, d and f), depending on the system mass and damping. As regards the maximum attainable responses $\left(A_{y m} / D, A_{x m} / D\right)$, Figure 3 shows a better comparison in the cross-flow VIV than in the in-line VIV, with both experimental and numerical responses providing $1.4<A_{y m} / D<1.75$. The numerical model apparently underestimates $A_{x m} / D$ although it predicts well the associated RMS values. These outcomes could be influenced by the temporal modulation of $A_{y} / D$ and $A_{x} / D$. To exemplify this aspect, experimental (dashed blue lines) and numerical (solid pink lines) time histories of $y$ and $x$ responses of KHL1 data with $V_{r}=10.9$ (Fig. 
3a and b) and KHL5 data with $V_{r}=11.7$ (Fig. 3c and d) are plotted in Fig. 4a-b and 4c-d, respectively. It is found that, in spite of the nearly-zero mean values of the time-varying $x$ (about 0.046 in Fig.4b and 0.013 in Fig. 4d), experimental in-line responses are seen to have a higher modulation when compared to the associated numerical ones. In contrast, both experimental and numerical y responses (Fig. 4a and c) are comparable, exhibiting a much less fluctuating signal.

In the case of $f^{*} \neq 1$, experimental and numerical comparisons of $A_{y} / D$ and $A_{x} / D$ are shown in Fig. 5. To also demonstrate the effect of empirical coefficients, two sets of numerical results are plotted: one based on $\Lambda_{x}=\Lambda_{y}=12$ (solid lines) and the other based on $\Lambda_{x}=\Lambda_{y}=15$ (dashed lines), while keeping other parameters unchanged. This change in $\Lambda_{x}$ and $\Lambda_{y}$ has been motivated by a possible variation of both lock-in ranges and ensuing amplitudes (Srinil and Zanganeh, 2012). With increasing $f^{*}$, some VIV behaviors are noticed experimentally. First, the in-line-only responses seem to disappear with increasing $f^{*}=1.6$ (Fig. 5d) and $f^{*}=1.85$ (Fig. 5f). This is in agreement with the numerical prediction. Secondly, both cross-flow and in-line responses in Fig. 5c and $5 \mathrm{~d}\left(f^{*}=1.6\right)$ and Fig. 5e and $5 f\left(f^{*}=1.85\right)$ reveal the flattening slopes of their upper branches with amplitudes starting from $V_{r} \approx 2.5$ and ending at $V_{r} \approx 12.5$. These amplitude profiles are qualitatively similar to the experimental results of Assi et al. (2009) with $f^{*}=1.93$.

Nevertheless, overall experimental results show $A_{y m} / D \approx 1.5$ and $A_{x m} / D \approx 0.5$, and the associated excitation ranges are quite comparable, in all $f^{*}$ cases. Given the similar values of $m^{*} \xi$, these imply the negligible effect of varying $f^{*}$ on the maximum response outcomes based on this pendulum-spring-cylinder system. With respect to numerical comparisons, the predicted $A_{y m} / D$ and $A_{x m} / D$ are found to be overestimated and the associated upper branches show higher slopes being typical for resonance diagrams. These reflect the difficulty in matching numerical and experimental results in which several coefficients control the dynamic responses and some of the influential parameters are variable, i.e. $\xi_{x} \neq \xi_{y}$. However, with a demonstrated small increment of $\Lambda_{x}$ and $\Lambda_{y}$, the qualitative prediction of lock-in ranges appears to be satisfactorily improved. Hence, values of $\Lambda_{x}=\Lambda_{y}=15$ are hereafter considered.

Next, it is of practical importance to carry out a sensitivity study on the numerical model in order to understand the influence of varying parameters on the 2-DOF VIV prediction and the dependence of the latter on $f^{*}$. To also capture possible qualitative and quantitative changes, the sensitivity analysis should be performed with respect to the parameters related to the greater $y$ response (Srinil and Zanganeh, 2012). By ways of examples, the geometrical coefficient $\alpha_{y}$ or $\beta_{y}$ is varied in the numerical simulations with $f^{*}=1.3,1.6$ and 2 . In each $f^{*}$ case, $m_{x}^{*}=m_{y}^{*}=1.4$ and 
the averaged $\xi_{x}=1.6 \%$ and $\xi_{y}=1 \%$ (based on KHL2-4 datasets) are assigned. Contour plots of $A_{y} / D$ and $A_{x} / D$ are displayed in Figs. 6 and 7 in the varying $\alpha_{y}$ and $\beta_{y}$ cases, respectively.

For each $f^{*}$, it is seen in Fig. 6 that $A_{y m} / D$ increases (Fig. 6a-c) whilst $A_{x m} / D$ decreases (Fig. 6d-f) as $\alpha_{y}$ increases, with the associated peaks locating at higher $V_{r}$ values. These reflect both the quantitative and qualitative influence of the cubic nonlinear stretching term which results in the bent-to-right response as $\alpha_{y} y^{3}$ becomes greater. On the other hand, it is found in Fig. 7 that, as $\beta_{y}$ increases, both $A_{y m} / D$ (Fig. 7a-c) and $A_{x m} / D$ (Fig. 7d-f) decrease; the associated peaks are slightly bent for lower $f^{*}$ (Fig. a and d) or nearly vertical for higher $f^{*}$ (Fig. 7b-c and 7e-f). These show the mostly quantitative effect of the geometric coupling $\beta_{y} y x^{2}$ term. Based on the above observations, the similar experimental response patterns with comparable $\xi_{y}$ and $\xi_{x}$ in Fig. 5 might be more influenced by the displacement coupling terms than the stretching nonlinearities. For this reason, a suitable new fixed $\beta_{y}$ value (e.g. 1.5) based on Fig. 7 may be suggested to improve the numerical quantitative comparison with experimental results in Fig. 5 whose $A_{y m} / D$ $\approx 1.5$ and $A_{x m} / D \approx 0.5$ in all $f^{*}$ cases. These sample contour plots might be applicable to a future prediction analysis once exact geometrical parameters are practically known.

Now, it is interesting to perform numerical and experimental comparisons of the time-varying orbital $x-y$ motions as well as phase angles because this information could shed some light on how the fluid-cylinder interaction affects the resulting vortex-shedding modes. Corresponding to KHL1-6 results in Figs. 3 and 5, the $x-y$ trajectory plots within several cycles of the oscillation are displayed in Fig. 8a with some chosen $V_{r}$. The normalized $x-y$ phase differences $\left(\theta_{x}-2 \theta_{y}\right) / \pi$ of KHL3 and KHL4 datasets are also exemplified in Fig. 8b. Depending on $f^{*}, m^{*}$ and $\xi$ (Table 1) and initial conditions in both numerical simulations and experiments, various characteristics of figure-of-eight trajectories appear with variable phase differences between $x$ and $y$ motions. In particular, the crescent shapes are evidenced in the experiments (see blue lines in Fig. 8a) with their tips pointing mostly downstream (all KHL datasets) and occasionally upstream (KHL3 and KHL4 for $V_{r}<10$ ). The former case justifies the use of system equations based on the model configuration in Fig. 2d. Similar orbital motions have been found in recent 2-DOF VIV experiments of rigid circular cylinders (Blevins and Coughran, 2009; Dahl et al., 2006; Dahl et al., 2010; Flemming and Williamson, 2005; Jauvtis and Williamson, 2004), and the present study confirms these studies with both experimental and numerical results.

It is worth noting that experimental orbital motions exhibit a high modulation feature of amplitudes whereby the oscillating cylinder does not follow the same path from cycle to cycle. 
This suggests a strong fluid-structure interaction effect associated with a 2:1 resonance during the test. On the contrary, numerical orbital motions are perfectly repeatable which justify the limit cycle character of the two pairs of coupled Duffing and van der Pol oscillators for which stable periodic solutions are attained. The numerical model is found to predict quite well overall qualitative behaviors of the figure-eight appearance due to the associated quadratic nonlinearities (Srinil and Zanganeh, 2012; Vandiver and Jong, 1987). The experimental (squares) and numerical (circles) comparisons of phase differences in Fig. 8b also reveal their good agreement in the range of about $8<V_{r}<14$ where response amplitudes are maximized (Fig. 5). By following the cylinder movement at the top of the figure of eight (Dahl et al., 2007), several figures of eight of KHL3 and KHL4 datasets can be defined as counterclockwise $\left(0<\theta_{x}-2 \theta_{y}<\pi / 2\right.$ and $3 \pi / 2<$ $\left.\theta_{x}-2 \theta_{y}<2 \pi\right)$ or clockwise $\left(\pi / 2<\theta_{x}-2 \theta_{y}<3 \pi / 2\right)$ paths. Yet, in the smaller-amplitude ranges of $V_{r}<8$ or $V_{r}>14$, a qualitative difference between experimental and numerical phase angles is still seen; this suggests possible use of the alternative configuration in Fig. 2b for the numerical model.

Experimental results in Fig. 8a suggest similar vortex formation patterns for KHL1, KHL5 and KHL6 with $f^{*}=1$ since the associated figures of eight are qualitatively similar in all $V_{r}$ cases. When increasing $f^{*}$ to be about 1.3 (KHL2), 1.6 (KHL3) and 1.9 (KHL4), the figure-eight orbits corresponding to some particular $V_{r}$ cases are noticed to be modified and these imply the possible change in the vortex formation patterns (Bao et al., 2012).

A comparison of experimental and numerical oscillation frequencies $\left(f_{o y}, f_{o x}\right)$ obtained from the amplitude responses within the main excitation ranges (Figs. 3 and 5) and normalized by the associated $f_{n y}$ is exemplified in Fig. 9 based on the selected KHL1 $\left(f^{*} \approx 1\right)$, KHL3 $\left(f^{*}=1.6\right)$ and KHL4 $\left(f^{*} \approx 1.9\right)$ data. Overall good qualitative agreement is appreciated, with $x$ (Fig. 9b, $\mathrm{d}$ and $\mathrm{f}$ ) and $y$ (Fig. 9a, c and e) frequency responses exhibiting their dual 2:1 resonances irrespective of the specified $f^{*}$. For the tested cylinder with low $m^{*}=1.4$, the oscillation frequencies of all dataset increase with increasing $V_{r}$ due to the decreasing value of the hydrodynamic added mass. This justifies the fundamental mechanism of 2-DOF VIV (Sarpkaya, 2004; Williamson and Govardhan, 2004).

\section{Experimental Comparisons with Other Studies}

It is of considerable theoretical and practical importance to understand the extent to which 2-DOF VIV results are sensitive to the various arrangements of test rigs and measurement procedures. A comparison is now presented between the results of the present study and those obtained by Dahl et al. (2006) at MIT using a very different test rig. The comparison between KHL (Table 1) and 
MIT (Table 2) experimental data are considered by categorizing the results into four groups depending on the comparable values of $f^{*}$ as follows:

a) KHL1, KHL5, KHL6 vs. MIT1, all with $f^{*} \approx 1$,

b) KHL2 vs. MIT2 and MIT3, all with the averaged $f^{*} \approx 1.3$,

c) KHL3 vs. MIT4 and MIT5, all with the averaged $f^{*} \approx 1.6$,

d) KHL4 vs. MIT6, all with $f^{*} \approx 1.9$.

Comparisons are made in terms of $A_{y} / D$ and $A_{x} / D$ diagrams (Fig. 10), the associated oscillation-to-natural frequency ratios $f_{o y} / f_{n y}$ and $f_{o x} / f_{n y}$ (Fig. 11) and the Griffin plots (Fig. 12) of peak amplitudes vs. the Skop-Griffin parameter $\mathrm{S}_{\mathrm{GX}}=2 \pi^{3} \mathrm{St}^{2} m_{x}^{*} \xi_{x}$ and $\mathrm{S}_{\mathrm{GY}}=2 \pi^{3} \mathrm{St}^{2} m_{y}^{*} \xi_{y}$ (Skop and Balasubramanian, 1997). Note that the value of $A_{y m} / D$ with MIT apparatus was limited to 1.35 (Dahl et al., 2006). $f_{o x}$ and $f_{o y}$ are the dominant oscillation frequencies obtained from the fast Fourier transform analysis of relevant response time histories.

With $f^{*} \approx 1$, overall response amplitudes of KHL1, KHL5, KHL6 and MIT1 data show a variation of $A_{y m} / D$ in the range of about 1.35-1.75 (Fig. 10a) and $A_{x m} / D$ in the range of about 0.40.8 (Fig. 10b). This disparity of peak responses may in part be due to the influence of variable $\xi_{y}$ and $\xi_{x}$ whose values are mostly $\xi_{y} \neq \xi_{x}$ (except MIT1). The KHL6 and MIT1 results with comparable $m^{*}$ (3.3-3.8) provide a good qualitative agreement with a similar lock-in range of $4<$ $V_{r}<12$ in which both $A_{y} / D$ and $A_{x} / D$ are simultaneously excited. Good qualitative agreements are also appreciated by the comparison of KHL1 and KHL5 data. In these lower $m^{*}=1.4$ cases, the lock-in range is noticed to be broader $\left(4<V_{r}<18\right)$. This influence of varying $m^{*}$ on the 2 DOF lock-in range has recently been highlighted by the experiments of Stappenbelt et al. (2007) and the numerical predictions of Srinil and Zanganeh (2012).

With the averaged $f^{*} \approx 1.3$ and $f^{*} \approx 1.6$, the comparison of KHL2, MIT2 and MIT3 data (Fig. 10c and d) and that of KHL3, MIT4 and MIT5 data (Fig. 10e and f) reveal their good qualitative agreement of $A_{y m} / D$ and $A_{x m} / D$ in a small range of about $1.35-1.5$ and 0.4-0.6, respectively. As previously mentioned, a difference in the lock-in range between KHL and MIT results is possibly due to their different $m^{*}$ values, apart from assigning whether $m_{x}^{*}=m_{y}^{*}(\mathrm{KHL})$ or $m_{x}^{*} \neq m_{y}^{*}(\mathrm{MIT})$. The effect of variable damping - which has been found to control the response amplitude rather than the lock-in range (Blevins and Coughran, 2009) - might in part again be responsible for the difference in response peaks as in the previous case of $f^{*}=1$. 
Both qualitative and quantitative differences are now realized when considering the results with the averaged $f^{*} \approx 1.9$. For the MIT6 data with $m_{x}^{*} \neq m_{y}^{*}$ and $\xi_{y} \neq \xi_{x}$, results reveal a two-peak cross-flow response (Fig. 10g) - similar to those reported in Sarpkaya (1995) with $f^{*}=2$ (although therein $m^{*}$ and $\xi$ were not reported) - with the two $A_{y m} / D \approx 1$ and 1.1 taking place at $V_{r}$ $\approx 5$ and 8 , respectively. Note that the MIT6 in-line response still exhibits a single peak of $A_{x} / D \approx$ 0.3 at $V_{r} \approx 8$ (Fig. 10h). These observations are in contrast with KHL4 results with $m_{x}^{*}=m_{y}^{*}$ and $\xi_{y} \neq \xi_{x}$ which show single-peak responses in both cross-flow and in-line responses. Recent numerical studies by Lucor and Triantafyllou (2008) have also found only single-peak responses with $m_{x}^{*}=m_{y}^{*}$ and $\xi_{y}=\xi_{x}$. Owing to the lower $m^{*}$ and $\xi$ of KHL4 data, the associated $A_{y m} / D \approx$ 1.3 (Fig. 10g) and $A_{x m} / D \approx 0.5$ (Fig. 10h) are greater and the associated lock-in range is wider of about $4<V_{r}<18$. These qualitatively justify the present experimental results.

In Fig. 11, overall comparisons of $f_{\text {oy }} / f_{n y}$ (a, c, e and g) and $f_{o x} / f_{n y}$ (b, d, f and h) plots highlight good correspondence between KHL and MIT results. In general, $f_{\text {oy }} / f_{n y}$ values vary from 0.5 to 2 and $f_{o x} / f_{n y}$ values vary from 1 to 3 , with increasing $V_{r}$. These imply the variation of hydrodynamic added mass caused by VIV; that is, its value is first positive when $f_{\text {oy }} / f_{n y}<1$ and $f_{o x} / f_{n y}<2$, being zero at $f_{o y} / f_{n y} \approx 1$ and $f_{o x} / f_{n y} \approx 2$, and then becoming negative when $f_{o y} / f_{n y}>1$ and $f_{o x} / f_{n y}>2$. Regardless of the assigned $f^{*}$, the $f_{o x} / f_{o y}$ values in Fig. 11 are nearly commensurable to 2:1 ratios in various $V_{r}$ cases. These confirm the existence of dual resonance conditions (Bao et al., 2012; Dahl et al., 2006; Dahl et al., 2010) and demonstrate the intrinsic quadratic relationships between in-line and cross-flow responses (Vandiver and Jong, 1987) corresponding to the various figure-of-eight orbital motions traced out in Fig. 8a in comparison with numerical prediction results. These outcomes also confirm other recent experimental results of circular cylinders undergoing 2-DOF VIV with $f^{*}=1$ (Blevins and Coughran, 2009; Jauvtis and Williamson, 2004; Sanchis et al., 2008).

Comparisons of various experimental 2-DOF VIV results (Blevins and Coughran, 2009; Dahl et al., 2006; Dahl et al., 2010; Stappenbelt et al., 2007) with $A_{y m} / D$ vs. $\mathrm{S}_{\mathrm{GY}}$ and $A_{x m} / D$ vs. $\mathrm{S}_{\mathrm{GX}}$ are now discussed through the Griffin plots in Fig. 12. Numerical prediction results with specified $m_{x}^{*}=m_{y}^{*}=m^{*}=1.4$ (lowest value from KHL data) and 5.7 (highest value from MIT data), and $f^{*}=$ 1 and 2 in each of these cases are also given. The numerical variation of $\mathrm{S}_{\mathrm{GY}}$ and $\mathrm{S}_{\mathrm{GX}}$ values (from 0.01 to 1 ) is performed by varying $\xi_{y}$ and $\xi_{x}$, respectively, with a small increment. A general qualitative agreement can be seen in Fig. 12 where both $A_{y m} / D$ and $A_{x m} / D$ decrease as 
$\mathrm{S}_{\mathrm{GY}}$ and $\mathrm{S}_{\mathrm{GX}}$ increase. However, for a specific $\mathrm{S}_{\mathrm{GY}}=\mathrm{S}_{\mathrm{GX}}$, experimental results with different values of $m^{*}, \xi$ and $f^{*}$ are scattered. The Re range might also play a role (Govardhan and Williamson, 2006) although the majority of the experimental results in Fig. 12 were based on the subcritical Re flows, except for some results in a supercritical Re range of Dahl et al. (2010). These imply how the combined mass-damping parameter fails to collapse different experimental 2-DOF VIV data. Numerical results also capture these quantitative effects, by also providing the approximate ranges of peak amplitudes and highlighting a possibly influential role of $f^{*}$. From a prediction viewpoint, both $A_{y m} / D$ and $A_{x m} / D$ increase as $m^{*}$ decreases; however, for a higher $m^{*}=$ 5.7 value, the variation of $A_{y m} / D$ is slightly influenced by the varying $f^{*}$. This observation is reminiscent of the experimental study of Jauvtis and Williamson (2004) where there was a slight influence on the cross-flow response with $m^{*}>6$ when comparing the results obtained between 1- and 2-DOF models. In contrast, numerical $A_{x m} / D$ values are found to be susceptible to any change of system $m^{*}, \xi$ and $f^{*}$ parameters. The above-mentioned discussion and comparisons deserve further experimental explorations before we could draw a firm conclusion on whether and how each - or the combination - of these parameters actually governs the 2-DOF VIV of circular cylinders.

\section{Discussion}

As 2-DOF VIV of a flexibly mounted circular cylinder depends on several system fluid-structure parameters in both cross-flow and in-line directions, it is a very challenging task to quantitatively match numerical prediction results to experimental measurements. One of the main reasons suggested is that some of the key variables were not assessed with sufficient confidence during the testing campaign. In particular, values of the structural damping in water - used as one of the inputs in the numerical model - were found to be sensitive to the initial displacement condition, the change of springs' stiffness and the apparatus arrangement leading to some repeatability issues in determining total and fluid-added damping from the free decay tests in water. This observation was in contrast to the measurements made in air for which the estimated damping was highly repeatable. As a consequence, the damping ratios $\xi_{x}$ and $\xi_{y}$ appeared variable and $\xi_{x}$ $\neq \xi_{y}$ when comparing and calibrating all datasets with different $f^{*}$. To overcome this constraint, an improved means to assess and control equivalent damping values in both directions (Klamo, 2009) or a systematic approach to measure system uncertainties (Hughes and Hase, 2010; Taylor, 1997) should be implemented. 
Another aspect deals with the difference between the numerical model idealization and the real experimental setup. For the sake of generality, the cylinder is theoretically postulated to be infinitely long such that the flow field might be approximated to be two-dimensional. However, during the experiment, the three-dimensional flow field along with the free surface around the oscillating cylinder with a finite length could play an influential role. Although the structurally geometric coupling terms associated with the movement of two pairs of springs have been accounted for in the numerical model, exact geometrical $\alpha_{x}, \alpha_{y}, \beta_{x}$ and $\beta_{y}$ values of the present experimental pendulum-spring-cylinder model are presently unknown. Some of these have been shown through a sensitivity study to produce a qualitative and quantitative effect on the prediction of both peak amplitudes and lock-in ranges (Figs. 6 and 7).

Nevertheless, after substantial parametric studies involving calibration and tuning of model empirical coefficients and geometric parameters with various experimental results, the numerical prediction model based on double Duffing-van der Pol oscillators is capable of predicting quite well several important qualitative features observed in the experimental 2-DOF VIV. These include (i) the pure in-line VIV lock-in ranges in the case of $f^{*}=1$ (Fig. 3) and their disappearances in the case of higher $f^{*}$ (Fig. 5), (ii) the two-dimensional lock-in ranges in all $f^{*}$ cases (Figs. 3 and 5), (iii) the response amplitude jump phenomena captured by system cubic nonlinearities (Fig. 3), (iv) various figure-of-eight trajectories representing periodically coupled $x-y$ motions associated with dual 2:1 resonances and captured by system quadratic nonlinearities (Figs. 8 and 9) and (v) the independent effect of $m^{*}, \xi$ and $f^{*}$ (Fig. 12). The hysteresis effect and the occurrence of critical mass whereby maximum cross-flow amplitudes exhibit an unbounded lock-in scenario have recently been shown in Srinil and Zanganeh (2012). With suitably specified coefficients, the possible occurrence of two-peak cross-flow response in the case of $f^{*}=2$ and $m_{x}^{*} \neq m_{y}^{*}$ has also been found during the parametric trials. Other insights into the effect of (displacement, velocity and acceleration) coupling terms in the wake oscillators, the appearance of smaller-amplitude higher harmonics in $x-y$ motions and the occurrence of chaotic VIV have been presented in Zanganeh and Srinil (2012). As far as model empirical coefficients in Eqs. (3)(6) are concerned, one may preliminarily suggest $\varepsilon_{y}$ based on Eq.(7), $\varepsilon_{x}=0.3,12<\Lambda_{x}=\Lambda_{y}<15$, and $\alpha_{x}=\alpha_{y}=\beta_{x}=\beta_{y}=0.7$, based on calibration with experimental results in the present study. Of course, the sensitivity analysis should also be performed, by limiting the number of considered cases with the only variation of $y$-related parameters. 
It is of practical importance to ascertain whether the variable $f^{*}$ influences the maximum attainable cross-flow/in-line amplitudes. Based on the range of $m^{*}, \xi$ and Re considered, our experimental results reveal the small or even negligible effect of varying $f^{*}$ on the maximum attainable amplitudes of 2-DOF VIV. This suggests that the model developed for a rigid cylinder with 2 DOF might be applicable to the analysis of a flexible cylinder vibrating in two directions with multi DOF and various $f^{*}$ through, e.g., a nonlinear modal expansion approach (Srinil, 2010, 2011; Srinil et al., 2009). However, the variation of $f *$ can influence the phase difference between cross-flow and in-line motions (Fig. 8b) which in turn may result in the change in the vortex shedding formation in the wake due to the fluid-structure coupling mechanism (Bao et al., 2012). These KHL observations are in good qualitative agreement with MIT results (Dahl et al., 2006) since both studies consider the similar ranges of $m^{*}, \xi, f^{*}$ and Re. It is noticed that the lock-in ranges of KHL data are greater than those of MIT data owing to the lower $m^{*}$ of the former (Fig. 10). Recent 2-D numerical simulation results of Bao et al. (2012) where $m_{x}^{*}=m_{y}^{*} \approx 2.55, \xi_{x}=\xi_{y}$ $=0$ showed comparable $A_{y m} / D$ and $A_{x m} / D$ with $f^{*}$ increasing from 1 to 1.5 . However, due to the very low $\operatorname{Re}=150$, their reported $A_{y m} / D$ and $A_{x m} / D$ values are quite small, being only about 0.60.9 and 0.05-0.25, respectively, when compared to KHL/MIT data in Fig. 10 and others in Fig. 12. Such difference in peak amplitudes in different Re data suggests a possible influence of Re on 2-DOF VIV, similar to what has been observed in the field tests of flexible cylinders (Swithenbank et al., 2008).

As a final remark, due to a finite length of the tank, it is presently unfeasible to perform a perfect sweeping test where a towing speed (i.e. $V$ ) is altered during a single run of the carriage. As this change (whether increasing or decreasing $V$ ) would require the time for the transient dynamics to die out in order to achieve the steady state responses (especially for large-amplitude 2-DOF vibrations in the neighbourhood of the hysteresis), the carriage would reach the end and be terminated before the cylinder steady-state response takes place. Hence, possible coexisting responses associated with the jump and hysteresis - as usually observed in a water flume facility (Jauvtis and Williamson, 2004) - were not ascertained in our towing tank tests although the proposed numerical model can capture such important behaviours (Srinil and Zanganeh, 2012). Depending on system parameters and initial conditions, the jump and hysteresis phenomena should be further experimentally investigated in the framework of a 2-DOF VIV of circular cylinder with variable $f^{*}$ and in a higher Re range. 


\section{Conclusions}

Experimental investigations of 2-DOF VIV of a flexibly mounted circular cylinder with a low equivalent mass ratio ( $m^{*}=1.4$ and 3.5 ) and variable in-line-to-cross-flow natural frequency ratio ( $f^{*} \approx 1,1.3,1.6,1.9$ ) have been performed in a water towing tank. The VIV experiments cover a sub-critical Re range of about $2 \times 10^{3}-5 \times 10^{4}$. A generalized numerical prediction model has also been investigated based on double Duffing-van der Pol (structure-wake) oscillators which can capture the structurally geometrical coupling and fluid-structure interaction effects through system cubic and quadratic nonlinearities. The model empirical coefficients have been calibrated based on new experimental results and parametric investigations, and their values have been suggested. Some important aspects in the 2-DOF VIV have been numerically captured which are in good qualitative agreement with experimental observations.

With low values of $m^{*}=1.4$ equally in both directions, the two-dimensional VIV excitation ranges have been experimentally found to be in a broad range of the reduced velocity parameter, $4<V_{r}<17.5$, with maximum attainable cross-flow and in-line amplitudes achieving high values of about 1.25-1.6 and 0.5-0.7, respectively, depending on the level and combination of the $x-y$ structural damping ratios in all $f^{*}$ cases. This damping parameter along with the two-directionally geometrical coupling coefficients might in part be responsible for the disparity of response amplitudes and the quantitative differences between experimental and numerical results, apart from the fact that actual three-dimensional features of the flow around the finite cylinder cannot be presently captured by the numerical model. As regards experimental comparisons, the present measurement results and MIT published data based on similar Re and mass-damping ratio ranges exhibit fairly good agreement with comparable response amplitudes, lock-in ranges and oscillation frequencies. However, there is no appearance of two-peak cross-flow response found in the present testing campaign as a result of the equivalent $m^{*}$ in the two motion directions. Regardless of the specified $f^{*}$ and overall hydro-geometric nonlinearities, various features of figure-of-eight orbital motions have been experimentally as well as numerically observed in a wide $V_{r}$ range. These evidence the fundamental characteristics of dual 2:1 resonances of coupled in-line/cross-flow VIV responses. The proposed numerical model is able to capture these dual resonances associated with quadratic nonlinearities in addition to the reasonable estimation of response amplitudes, lock-in ranges and oscillation frequencies.

More experimental and computational fluid dynamics studies which assess and control the equivalence of system fluid-structure parameters in different directions with reduced uncertainty are needed to improve the model empirical coefficients and capability in effectively matching 
numerical predictions to experimental outcomes. These should be furnished by the identification of vortex formation patterns in the cylinder wake using the flow visualization technique such as the particle image velocimetry.

\section{Acknowledgements}

The authors are grateful to the SORSAS and Early Career Researcher International Exchange Awards supported by the University of Strathclyde and Scottish Funding Council (via GRPeSFC). They also wish to thank technicians at the Kelvin Hydrodynamics Laboratory for the experimental setup and measurement support, as well as the reviewers for their helpful comments.

\section{References}

Assi, G.R.S., Bearman, P.W., Kitney, N., 2009. Low drag solutions for suppressing vortex-induced vibration of circular cylinders. Journal of Fluids and Structures 25 (4), 666-675.

Assi, G.R.S., Srinil, N., Freire, C.M., Korkischko, I., 2012. Experimental investigation of the vortexinduced vibration of a curved cylinder, The 31st International Conference on Ocean, Offshore and Arctic Engineering, pp. OMAE2012-83016.

Bao, Y., Huang, C., Zhou, D., Tu, J., Han, Z., 2012. Two-degree-of-freedom flow-induced vibrations on isolated and tandem cylinders with varying natural frequency ratios. Journal of Fluids and Structures 35, 50-75.

Bearman, P.W., 2011. Circular cylinder wakes and vortex-induced vibrations. Journal of Fluids and Structures 27 (5-6), 648-658.

Bishop, R.E.D., Hassan, A.Y., 1964. The lift and drag forces on a circular cylinder oscillating in a flowing fluid, Proceedings of the Royal Society of London, pp. 51-75.

Blevins, R.D., 1990. Flow-Induced Vibrations. Van Nostrand Reinhold, New York.

Blevins, R.D., Coughran, C.S., 2009. Experimental investigation of vortex-induced vibration in one and two dimensions with variable mass, damping, and Reynolds number. Journal of Fluids Engineering 131 (10), 101202-101207.

Currie, I.G., Turnbull, D.H., 1987. Streamwise oscillations of cylinders near the critical Reynolds number. Journal of Fluids and Structures 1 (2), 185-196.

Dahl, J.M., Hover, F.S., Triantafyllou, M.S., 2006. Two-degree-of-freedom vortex-induced vibrations using a force assisted apparatus. Journal of Fluids and Structures 22 (6-7), 807-818.

Dahl, J.M., Hover, F.S., Triantafyllou, M.S., Dong, S., Karniadakis, G.E., 2007. Resonant Vibrations of Bluff Bodies Cause Multivortex Shedding and High Frequency Forces. Physical Review Letters 99 (14), 144503. 
Dahl, J.M., Hover, F.S., Triantafyllou, M.S., Oakley, O.H., 2010. Dual resonance in vortex-induced vibrations at subcritical and supercritical Reynolds numbers, Journal of Fluid Mechanics, pp. 395-424.

Facchinetti, M.L., de Langre, E., Biolley, F., 2004. Coupling of structure and wake oscillators in vortexinduced vibrations. Journal of Fluids and Structures 19 (2), 123-140.

Flemming, F., Williamson, C.H.K., 2005. Vortex-induced vibrations of a pivoted cylinder. Journal of Fluid Mechanics 522, 215-252.

Gabbai, R.D., Benaroya, H., 2005. An overview of modeling and experiments of vortex-induced vibration of circular cylinders. Journal of Sound and Vibration 282 (3-5), 575-616.

Govardhan, R., Williamson, C., 2006. Defining the 'modified Griffin plot' in vortex-induced vibration: revealing the effect of Reynolds number using controlled damping. Journal of Fluid Mechanics 561, 147180.

Hansen, E., Bryndum, M., Mayer, S., 2002. Interaction of in-line and cross-flow vortex-induced vibrations in risers, The 21st International Conference on Offshore Mechanics and Arctic Engineering, Oslo, pp. OMAE2002-28303.

Hughes, I.G., Hase, T.P.A., 2010. Measurements and their Uncertainties: A Practical Guide to Modern Error Analysis. Oxford.

Jauvtis, N., Williamson, C.H.K., 2003. Vortex-induced vibration of a cylinder with two degrees of freedom. Journal of Fluids and Structures 17 (7), 1035-1042.

Jauvtis, N., Williamson, C.H.K., 2004. The effect of two degrees of freedom on vortex-induced vibration at low mass and damping. Journal of Fluid Mechanics 509, 23-62.

Jeon, D., Gharib, M., 2001. On circular cylinders undergoing two-degree-of-freedom forced motions. Journal of Fluids and Structures 15 (3-4), 533-541.

Khalak, A., Williamson, C.H.K., 1999. Motions, forces and mode transitions in vortex-induced vibrations at low mass-damping. Journal of Fluids and Structures 13 (7-8), 813-851.

Kim, W.J., Perkins, N.C., 2002. Two-dimensional vortex-induced vibration of cable suspensions. Journal of Fluids and Structures 16 (2), 229-245.

Klamo, J., 2009. The application of controlled variable magnetic eddy current damping to the study of vortex-induced vibrations. Experiments in Fluids 47 (2), 357-367.

Laneville, A., 2006. On vortex-induced vibrations of cylinders describing $\mathrm{X}-\mathrm{Y}$ trajectories. Journal of Fluids and Structures 22 (6-7), 773-782.

Lucor, D., Triantafyllou, M.S., 2008. Parametric study of a two degree-of-freedom cylinder subject to vortex-induced vibrations. Journal of Fluids and Structures 24 (8), 1284-1293.

Moe, G., Wu, Z.J., 1990. The lift force on a cylinder vibrating in a current. Journal of Offshore Mechanics and Arctic Engineering 112 (4), 297-303.

Morse, T.L., Govardhan, R.N., Williamson, C.H.K., 2008. The effect of end conditions on the vortexinduced vibration of cylinders. Journal of Fluids and Structures 24 (8), 1227-1239. 
Nayfeh, A.H., 1993. Introduction to perturbation techniques. Wiley, New York.

Pesce, C., Fujarra, A., 2005. The super-upper branch VIV response of flexible cylinders, International Conference on Bluff Body Wakes and Vortex-Induced Vibrations, Santorini.

Sanchis, A., Sælevik, G., Grue, J., 2008. Two-degree-of-freedom vortex-induced vibrations of a springmounted rigid cylinder with low mass ratio. Journal of Fluids and Structures 24 (6), 907-919.

Sarpkaya, T., 1995. Hydrodynamic damping, flow-induced oscillations, and biharmonic response. Journal of Offshore Mechanics and Arctic Engineering 117 (4), 232-238.

Sarpkaya, T., 2004. A critical review of the intrinsic nature of vortex-induced vibrations. Journal of Fluids and Structures 19 (4), 389-447.

Skop, R.A., Balasubramanian, S., 1997. A new twist on an old model for vortex-excited vibrations. Journal of Fluids and Structures 11 (4), 395-412.

Srinil, N., 2010. Multi-mode interactions in vortex-induced vibrations of flexible curved/straight structures with geometric nonlinearities. Journal of Fluids and Structures 26 (7-8), 1098-1122.

Srinil, N., 2011. Analysis and prediction of vortex-induced vibrations of variable-tension vertical risers in linearly sheared currents. Applied Ocean Research 33 (1), 41-53.

Srinil, N., Rega, G., 2007. Two-to-one resonant multi-modal dynamics of horizontal/inclined cables. Part II: Internal resonance activation, reduced-order models and nonlinear normal modes. Nonlinear Dynamics 48 (3), 253-274.

Srinil, N., Rega, G., Chucheepsakul, S., 2007. Two-to-one resonant multi-modal dynamics of horizontal/inclined cables. Part I: Theoretical formulation and model validation. Nonlinear Dynamics 48 (3), 231-252.

Srinil, N., Wiercigroch, M., O’Brien, P., 2009. Reduced-order modelling of vortex-induced vibration of catenary riser. Ocean Engineering 36 (17-18), 1404-1414.

Srinil, N., Zanganeh, H., 2012. Modelling of coupled cross-flow/in-line vortex-induced vibrations using double Duffing and van der Pol oscillators. Ocean Engineering 53, 83-97.

Srinil, N., Zanganeh, H., 2013. Corrigendum to "Modelling of coupled cross-flow/in-line vortex-induced vibrations using double Duffing and van der Pol oscillators” [Ocean Eng. 53 (2012) 83-97]. Ocean Engineering 57 (0), 256.

Stappenbelt, B., 2010. Vortex-induced motion of nonlinear offshore structures: A study of catenary moored system fluid-elastic instability. Lambert Academic Publishing.

Stappenbelt, B., Lalji, F., Tan, G., 2007. Low mass ratio vortex-induced motion, The 16th Australasian Fluid Mechanics Conference, Gold Coast, Australia,, pp. 1491-1497.

Stappenbelt, B., O'Neill, L., 2007. Vortex-induced vibration of cylindrical structures with low mass ratio, The 17th International Offshore and Polar Engineering Conference, Lisbon.

Sumer, B.M., Fredsoe, J., 2006. Hydrodynamics Around Cylindrical Structures. World Scientific. 
Swithenbank, S.B., Vandiver, J.K., Larsen, C.M., Lie, H., 2008. Reynolds number dependence of flexible cylinder VIV response data, The 27th International Conference on Offshore Mechanics and Arctic Engineering, pp. OMAE2008-57045.

Taylor, J.R., 1997. An Introduction to Error Analysis: The Study of Uncertainties in Physical Measurements. University Science Books.

Trim, A.D., Braaten, H., Lie, H., Tognarelli, M.A., 2005. Experimental investigation of vortex-induced vibration of long marine risers. Journal of Fluids and Structures 21 (3), 335-361.

Vandiver, J.K., Jong, J.Y., 1987. The relationship between in-line and cross-flow vortex-induced vibration of cylinders. Journal of Fluids and Structures 1 (4), 381-399.

Wang, X.Q., So, R.M.C., Chan, K.T., 2003. A non-linear fluid force model for vortex-induced vibration of an elastic cylinder. Journal of Sound and Vibration 260 (2), 287-305.

Williamson, C.H.K., Govardhan, R., 2004. Vortex-induced vibrations. Annual Review of Fluid Mechanics 36, 413-455.

Williamson, C.H.K., Jauvtis, N., 2004. A high-amplitude 2T mode of vortex-induced vibration for a light body in XY motion. European Journal of Mechanics - B/Fluids 23 (1), 107-114.

Zanganeh, H., Srinil, N., 2012. Interaction of wake and structure in two-dimensional vortex-induced vibrations, International Conference on Advances and Challenges in Marine Noise and Vibration, Glasgow, pp. 177-188.

Zhao, M., Cheng, L., 2011. Numerical simulation of two-degree-of-freedom vortex-induced vibration of a circular cylinder close to a plane boundary. Journal of Fluids and Structures 27 (7), 1097-1110. 


\section{TABLE Captions}

Table 1 KHL experimental data with variable $m^{*}, \xi$ and $f^{*}$

Table 2 MIT experimental data with variable $m^{*}$, $\xi$ and $f^{*}$

\section{FIGURE Captions}

Figure 1 Experimental model of a flexibly mounted circular cylinder undergoing 2-DOF VIV

Figure 2 A schematic numerical model of a spring-supported circular cylinder undergoing 2DOF VIV (a) due to effective lift/drag forces exerted on the oscillating cylinder (b) or (d): sample figure-of-eight trajectories (c) and (e) based on model in (b) and (d), respectively.

Figure 3 Comparison of numerical (lines) and experimental (symbols) cross-flow and in-line amplitude responses based on KHL data with $f^{*}=1$ : blue lines and squares (pink lines and circles) denote maximum (RMS) values; dashed lines denote numerical response jumps.

Figure 4 Comparison of numerical (pink solid lines) and experimental (blue dashed lines) cross-flow (a, c) and in-line (b, d) time histories: KHL1 data with $V_{r}=10.9$ (a, b) and KHL5 data with $V_{r}=11.7$ (c, d).

Figure 5 Comparison of numerical (lines) and experimental (symbols) cross-flow and in-line amplitude responses based on KHL data with $f^{*} \neq 1$ : blue lines and squares (pink lines and circles) denote maximum (RMS) values; dashed (solid) lines with $\Lambda_{x}=\Lambda_{y}=15$ (12).

Figure 6 Sensitivity analysis showing the influence of geometrical parameter $\alpha_{y}$ on cross-flow (a-c) and in-line (d-f) amplitude responses: $f^{*}=1.3$ (a, d), $f^{*}=1.6$ (b, e), $f^{*}=2$ (c, f).

Figure 7 Sensitivity analysis showing the influence of geometrical parameter $\beta_{y}$ on cross-flow (a-c) and in-line (d-f) amplitude responses: $f^{*}=1.3$ (a, d), $f^{*}=1.6$ (b, e), $f^{*}=2$ (c, f).

Figure 8 (a) Comparison of numerical (red lines) and experimental (blue lines) $x-y$ trajectories based on KHL datasets with variable $f^{*}$; (b) comparison of numerical (circles) and experimental (squares) $x-y$ phase differences for KHL3 (filled symbols) and KHL4 (open symbols) dataset.

Figure 9 Comparison of experimental (circles) and numerical (squares) cross-flow/in-line oscillation frequencies as function of $V_{r}$ for selected KHL datasets with variable $f^{*}$.

Figure 10 Experimental comparisons of cross-flow and in-line amplitudes between KHL and MIT data with variable $f^{*}$.

Figure 11 Experimental comparisons of normalized cross-flow and in-line oscillation frequencies between KHL and MIT data with variable $f^{*}$.

Figure 12 Griffin plots of maximum attainable cross-flow and in-line amplitudes based on several 2-DOF VIV experimental (symbols) and numerical prediction (lines) results. 
Table 1

\begin{tabular}{|c|c|c|c|c|c|c|c|}
\hline Dataset & $f_{n y}(\mathrm{~Hz})$ & $f_{n x}(\mathrm{~Hz})$ & $\xi_{y}(\%)$ & $\xi_{x}(\%)$ & $m_{y}^{*}$ & $m_{x}^{*}$ & $f^{*}$ \\
\hline KHL1 & 0.312 & 0.316 & 1.0 & 4.7 & 1.4 & 1.4 & 1.01 \\
\hline KHL2 & 0.218 & 0.281 & 1.5 & 1.0 & 1.4 & 1.4 & 1.29 \\
\hline KHL3 & 0.262 & 0.419 & 1.6 & 1.0 & 1.4 & 1.4 & 1.60 \\
\hline KHL4 & 0.203 & 0.376 & 1.8 & 1.2 & 1.4 & 1.4 & 1.85 \\
\hline KHL5 & 0.192 & 0.192 & 2.0 & 3.1 & 1.4 & 1.4 & 1.00 \\
\hline KHL6 & 0.223 & 0.223 & 1.5 & 2.3 & 3.5 & 3.5 & 1.00 \\
\hline
\end{tabular}

$\underline{\text { Table } 2}$

\begin{tabular}{|c|c|c|c|c|c|c|c|}
\hline Dataset & $f_{n y}(\mathrm{~Hz})$ & $f_{n x}(\mathrm{~Hz})$ & $\xi_{y}(\%)$ & $\xi_{x}(\%)$ & $m_{y}^{*}$ & $m_{x}^{*}$ & $f^{*}$ \\
\hline MIT1 & 0.715 & 0.715 & 2.2 & 2.2 & 3.8 & 3.3 & 1.00 \\
\hline MIT2 & 0.799 & 0.975 & 1.3 & 1.7 & 3.9 & 3.8 & 1.22 \\
\hline MIT3 & 0.894 & 1.225 & 1.1 & 2.5 & 3.9 & 3.7 & 1.37 \\
\hline MIT4 & 0.977 & 1.485 & 1.6 & 3.2 & 4.0 & 3.6 & 1.52 \\
\hline MIT5 & 0.698 & 1.166 & 2.6 & 2.9 & 5.5 & 5.3 & 1.67 \\
\hline MIT6 & 0.704 & 1.338 & 6.2 & 2.5 & 5.7 & 5.0 & 1.90 \\
\hline
\end{tabular}



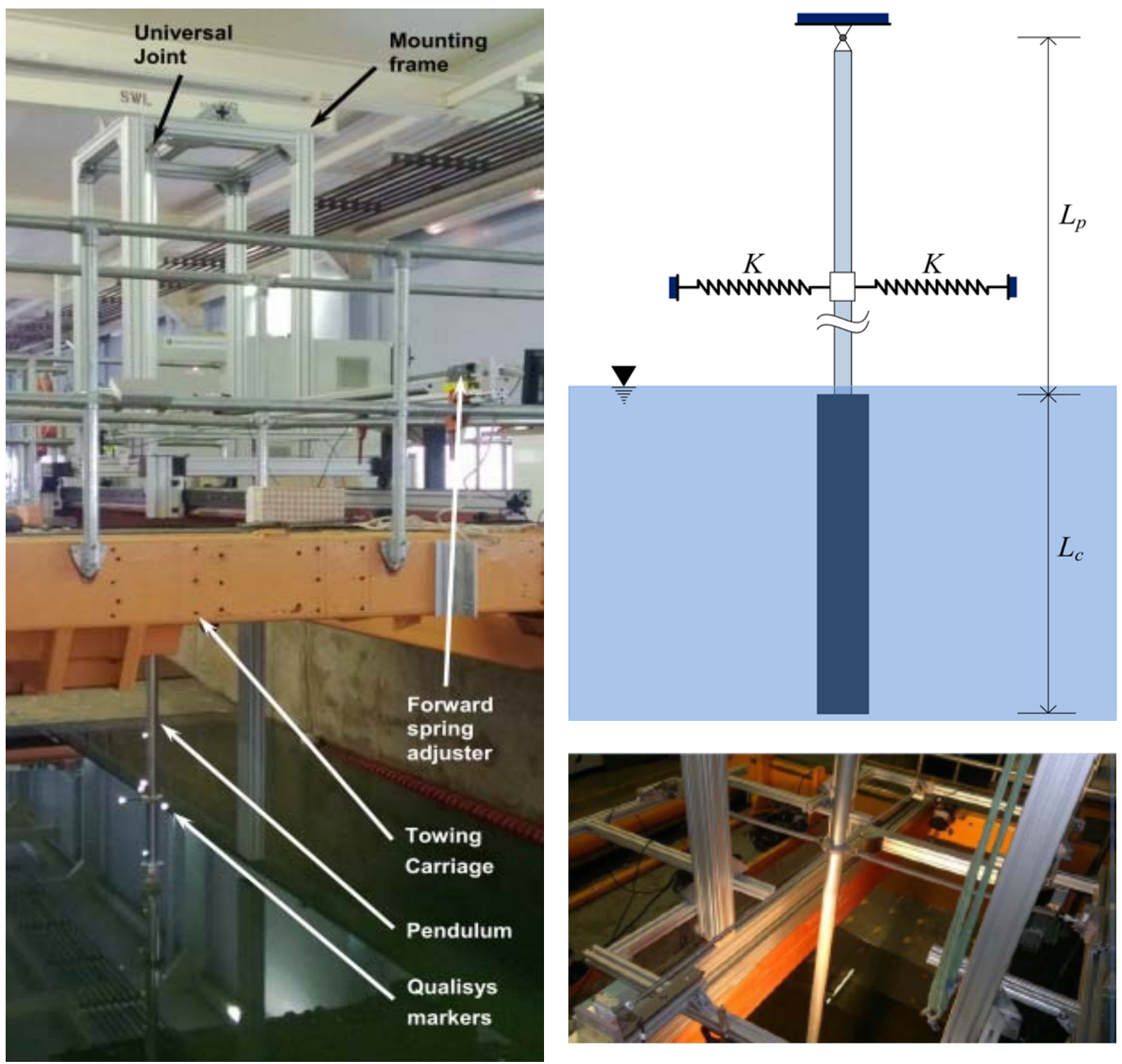

Figure 1 


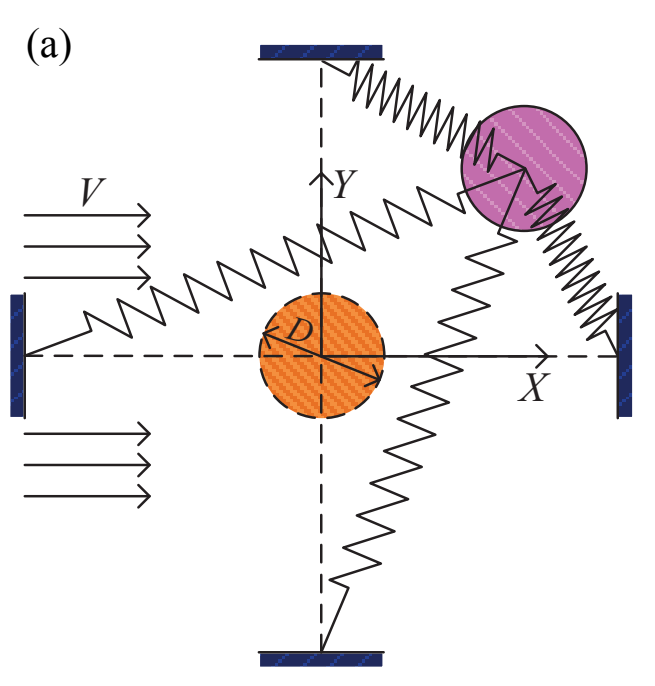

(b)

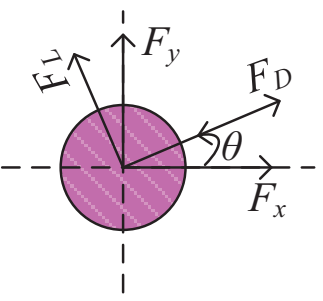

(c)

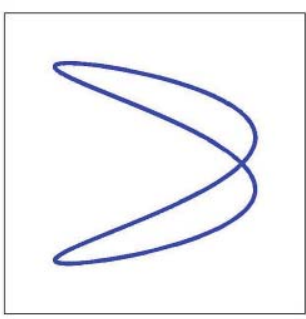

(d)

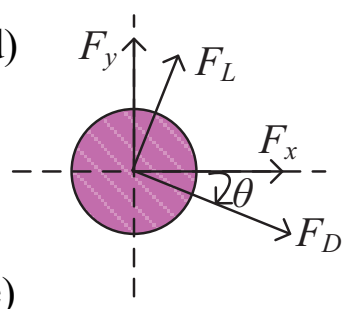

(e)

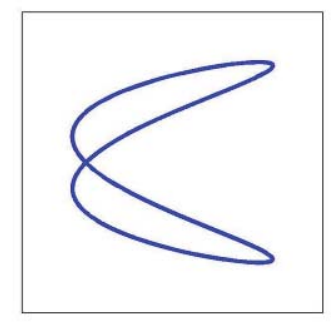

Figure 2 

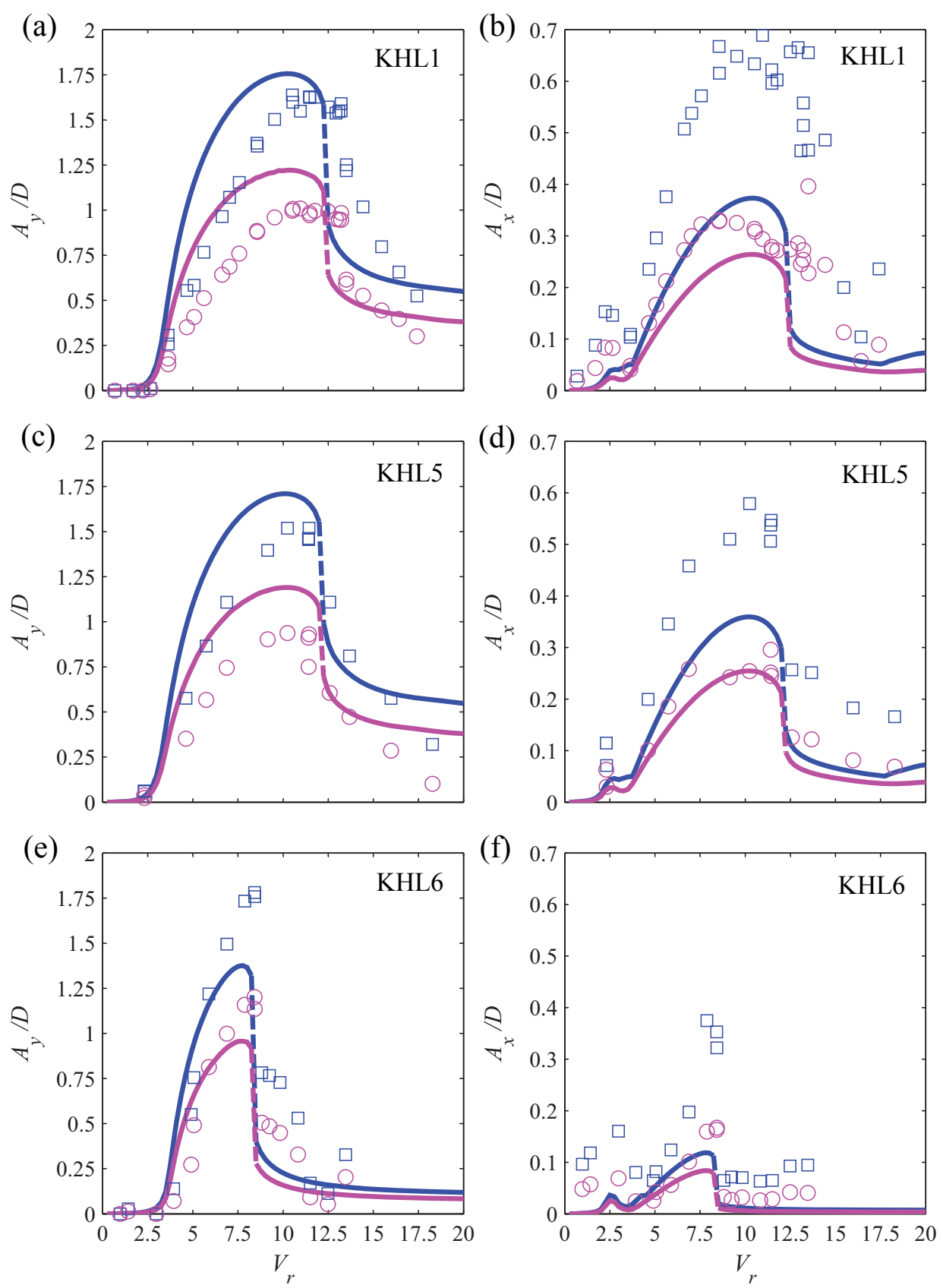

Figure 3 
(a)

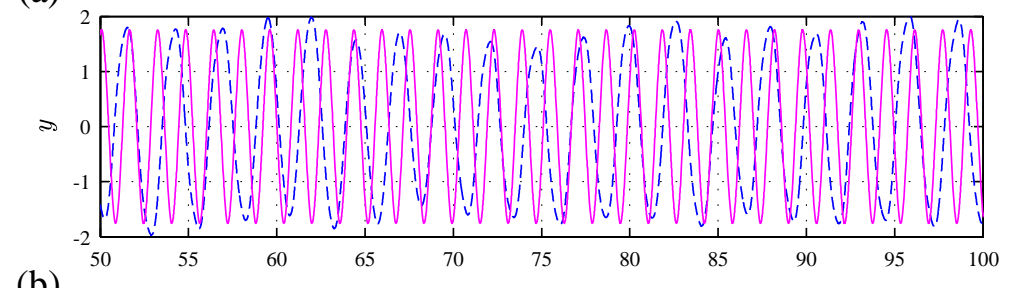

(b)

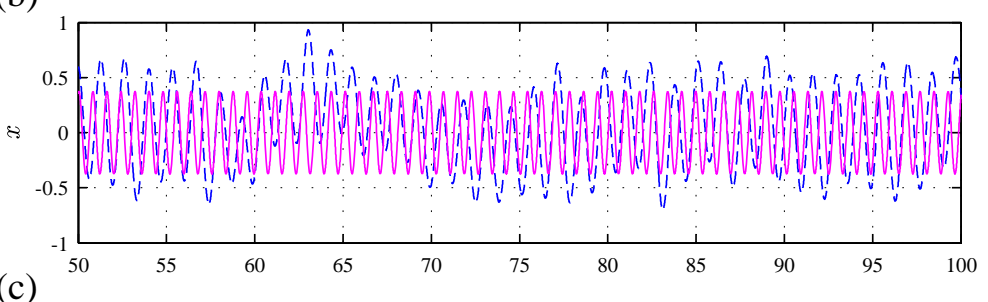

(c)

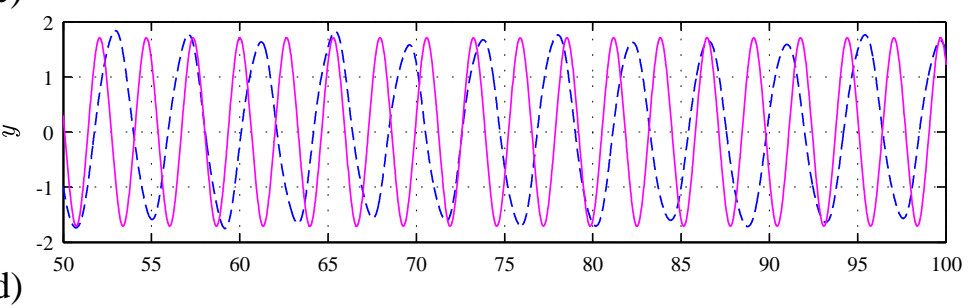

(d)

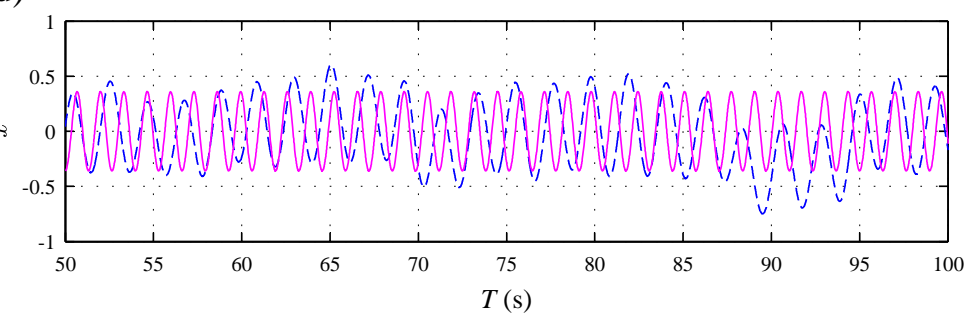

Figure 4 

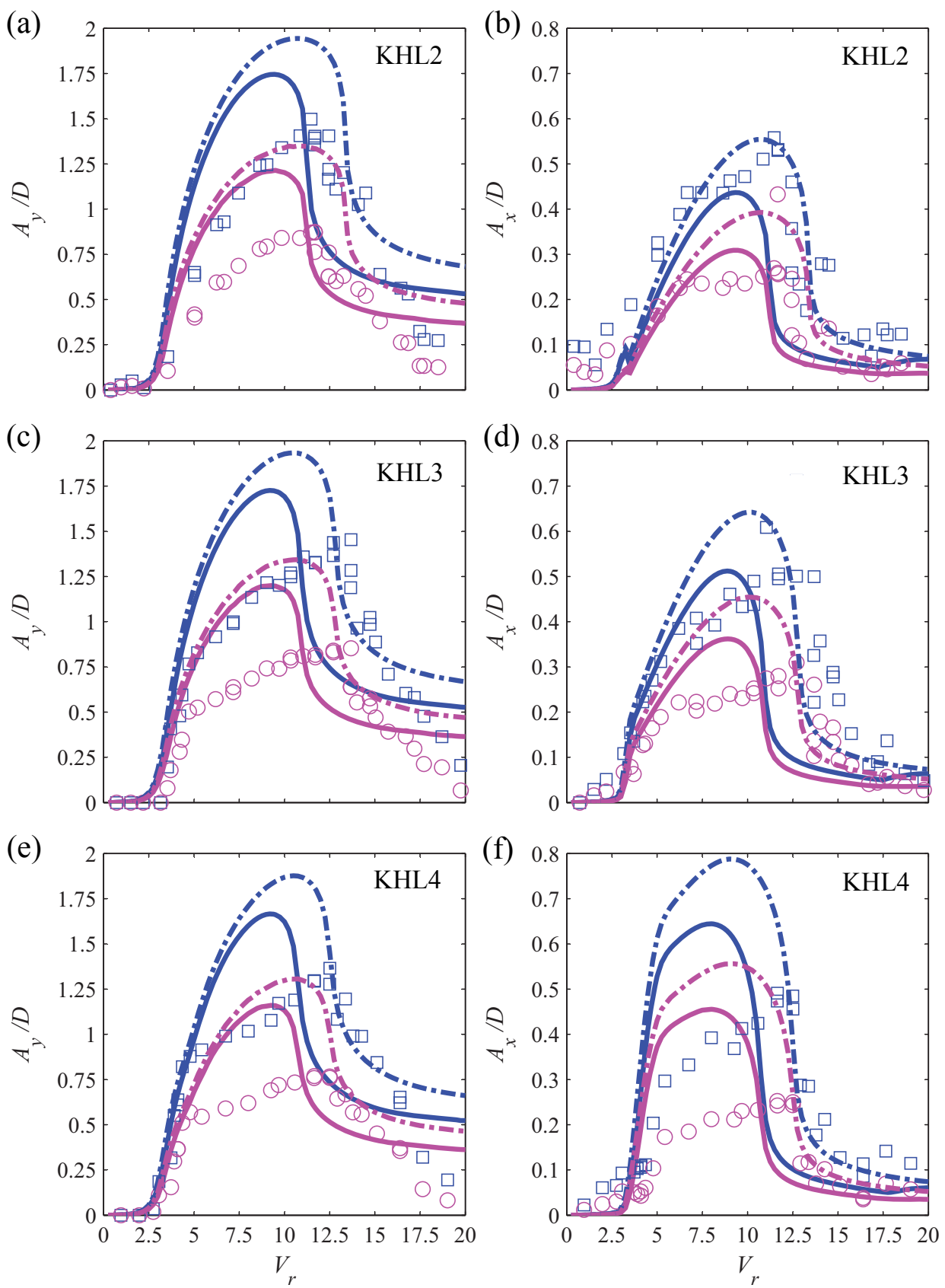

Figure 5 

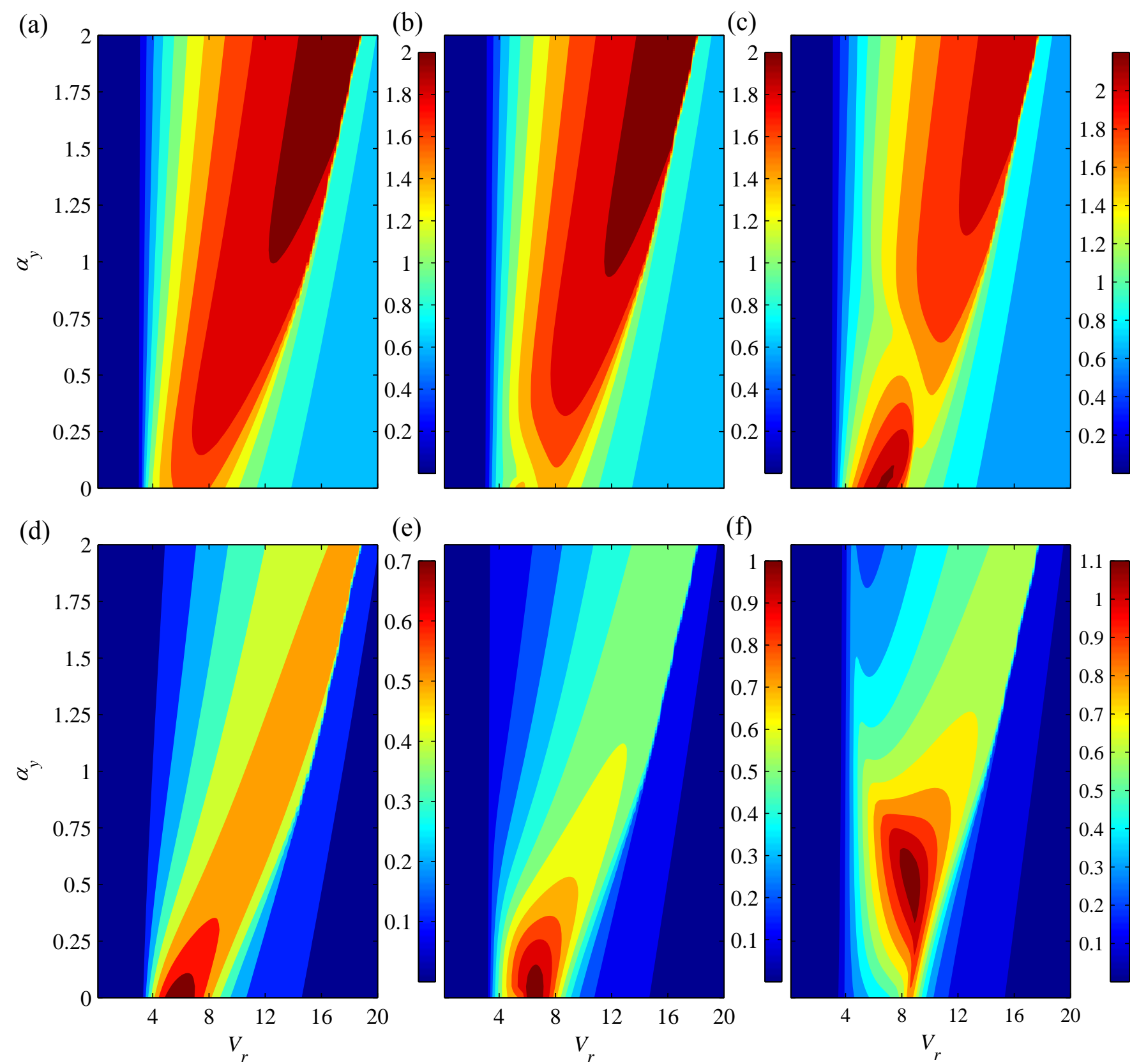

Figure 6 

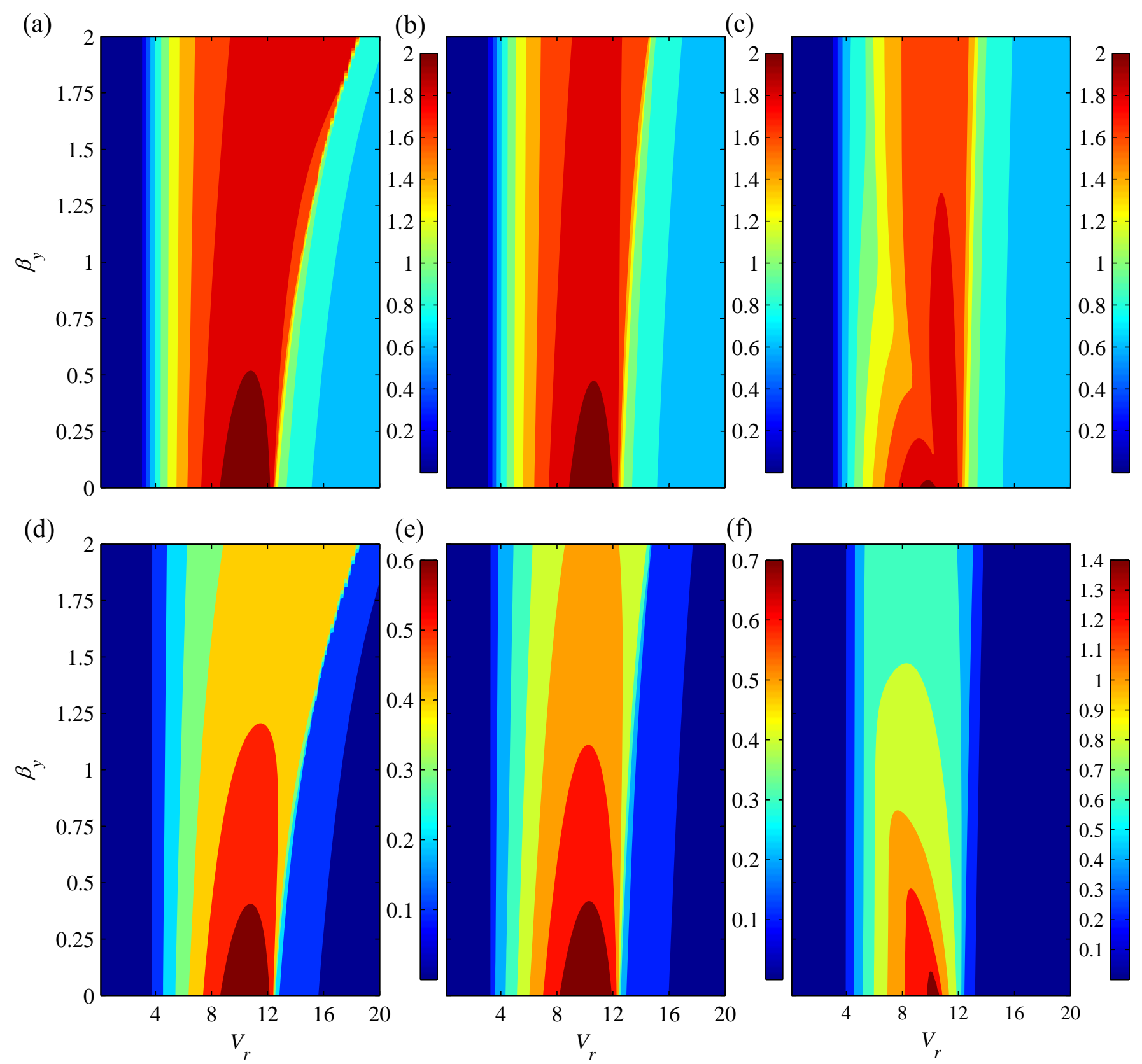

Figure 7 

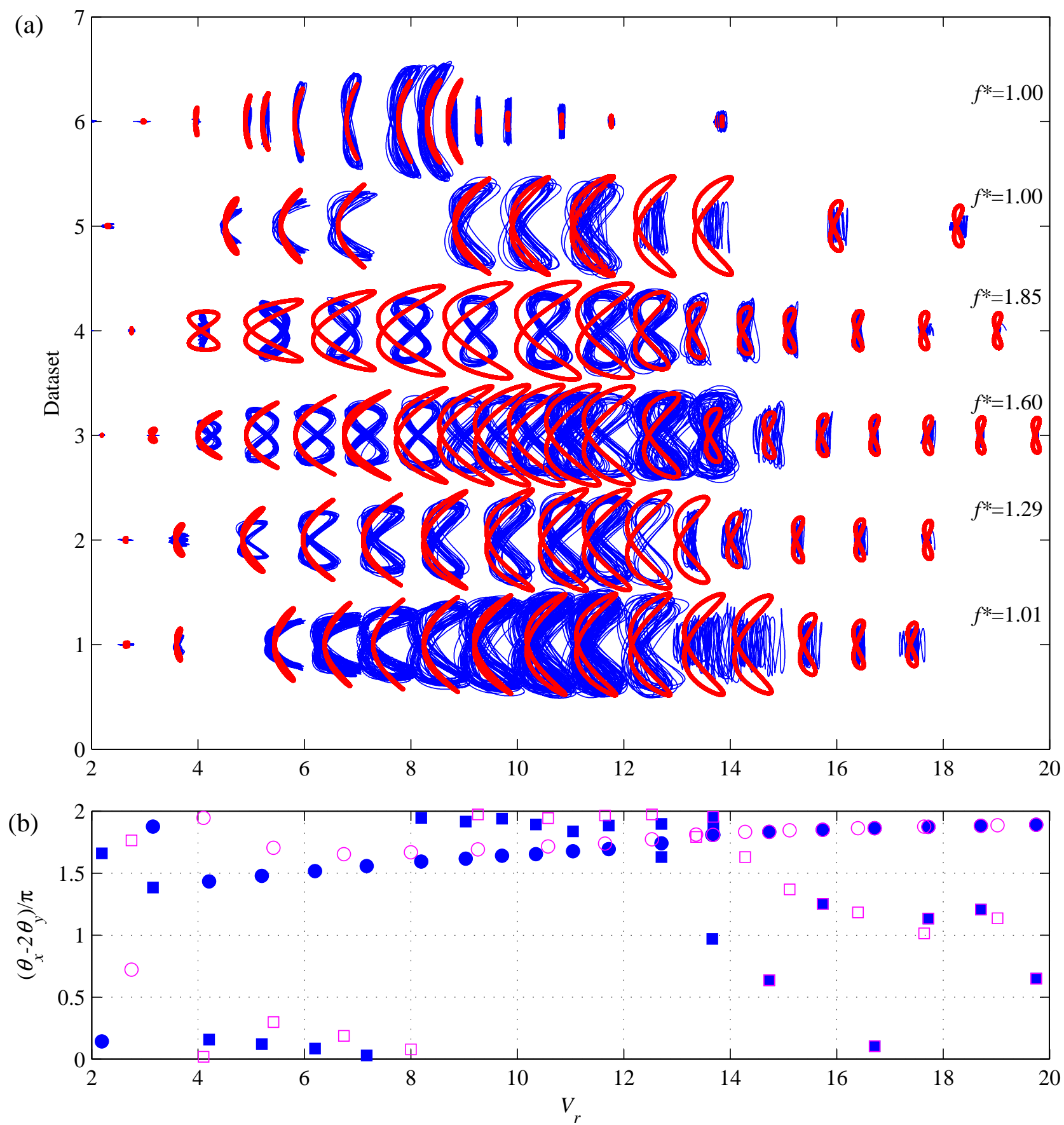

Figure 8 
(a)

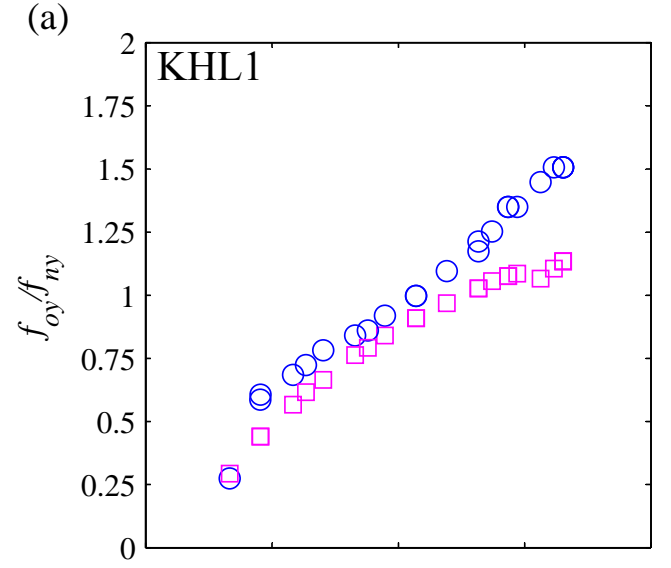

(c)

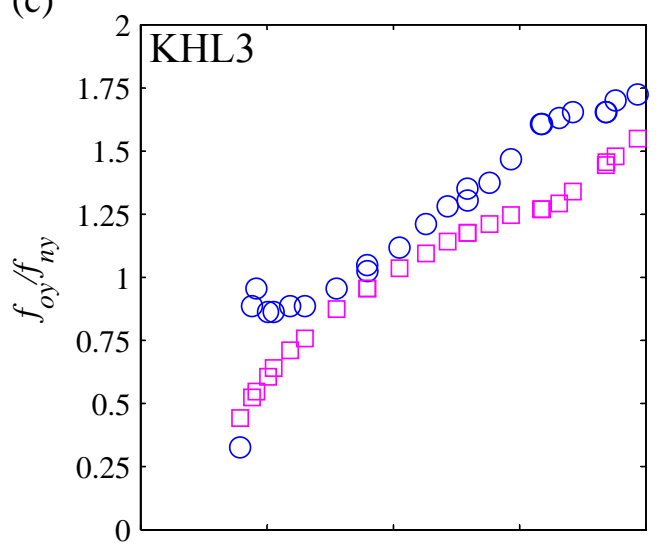

(e)

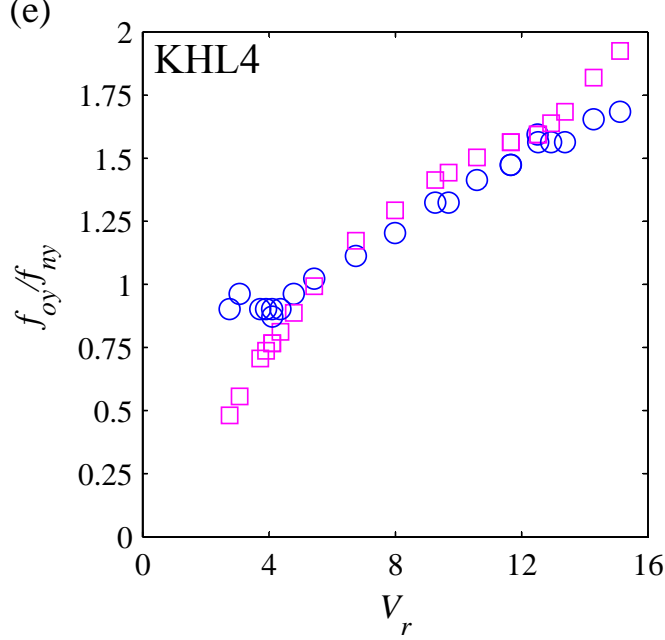

(b)

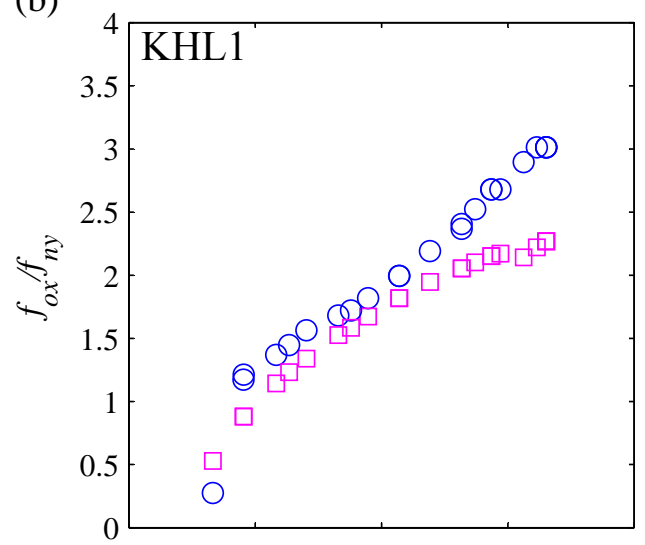

(d)

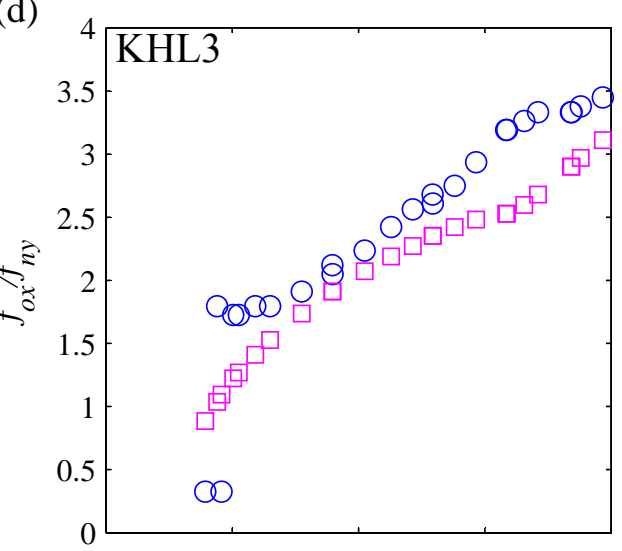

(f)

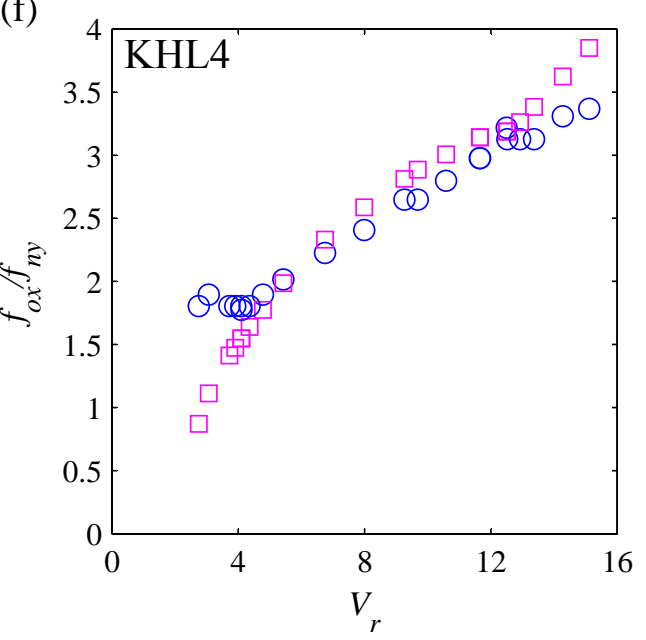

Figure 9 
(a)

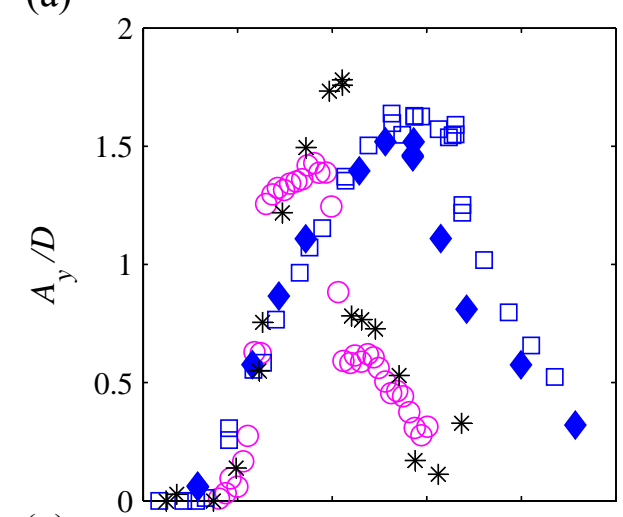

(c)

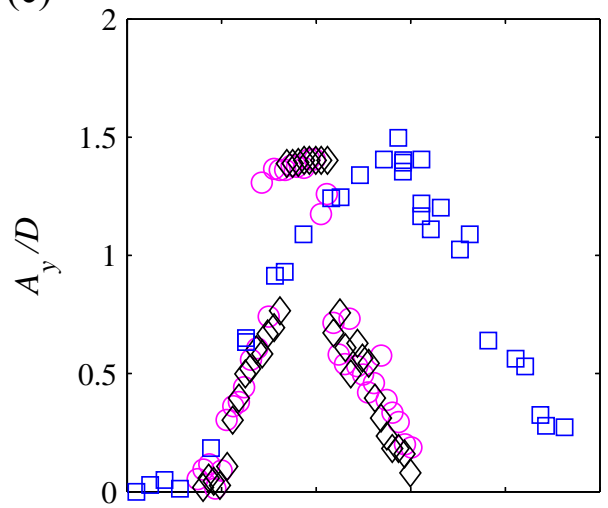

(e)
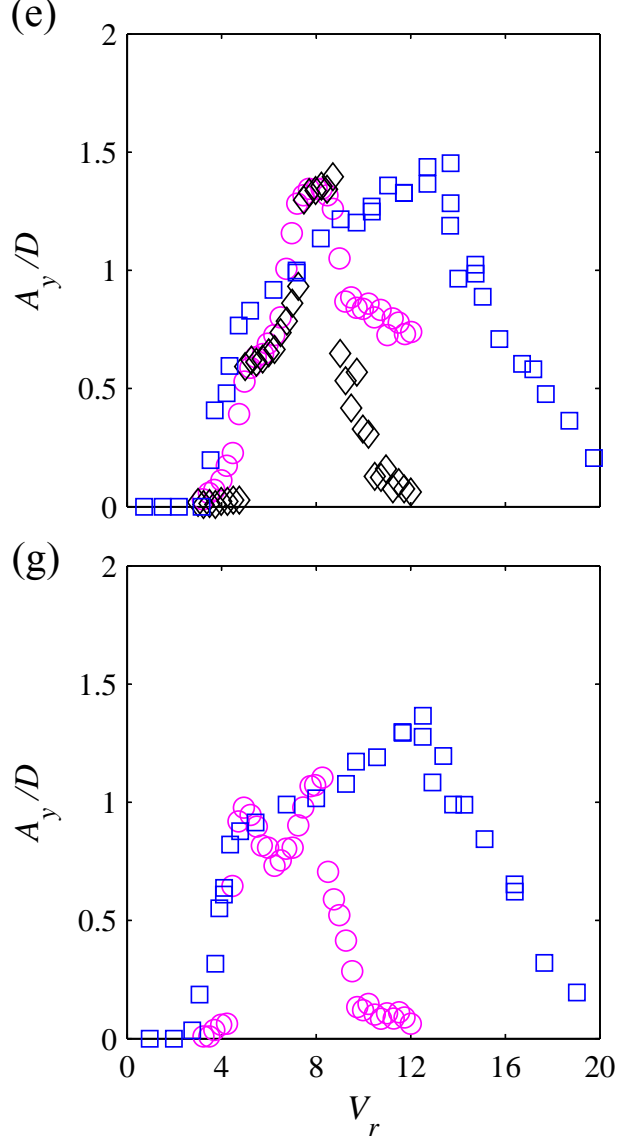

(b)

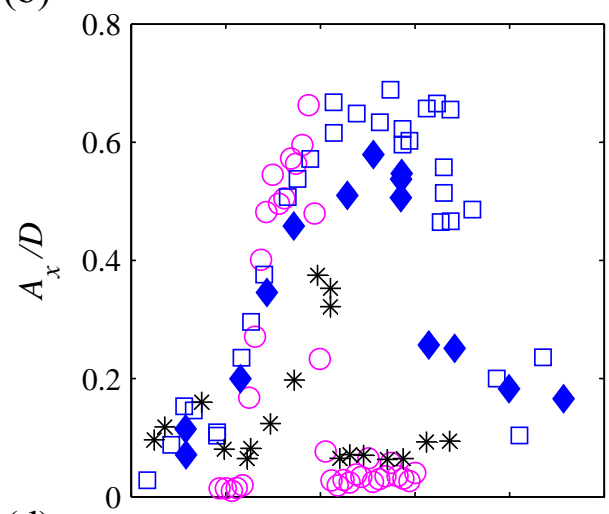

(d)

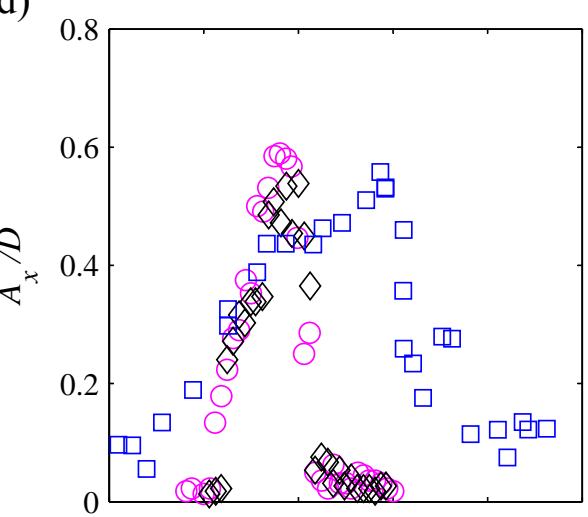

(f)
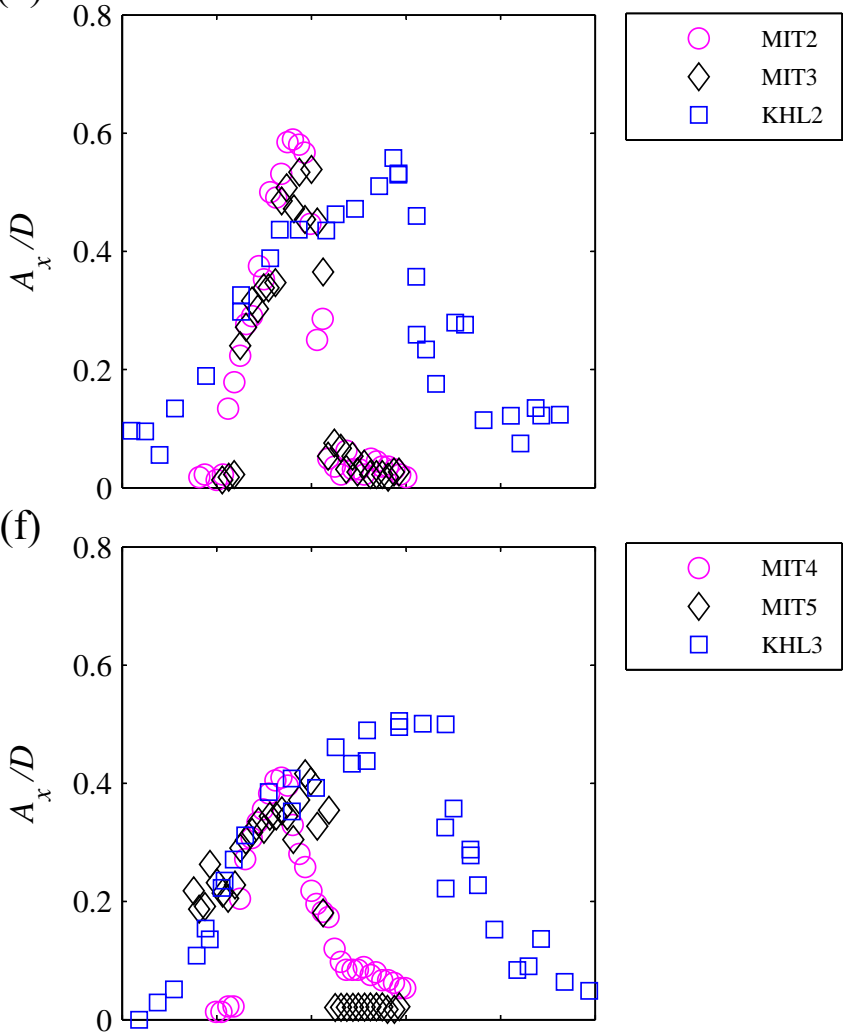

(h)

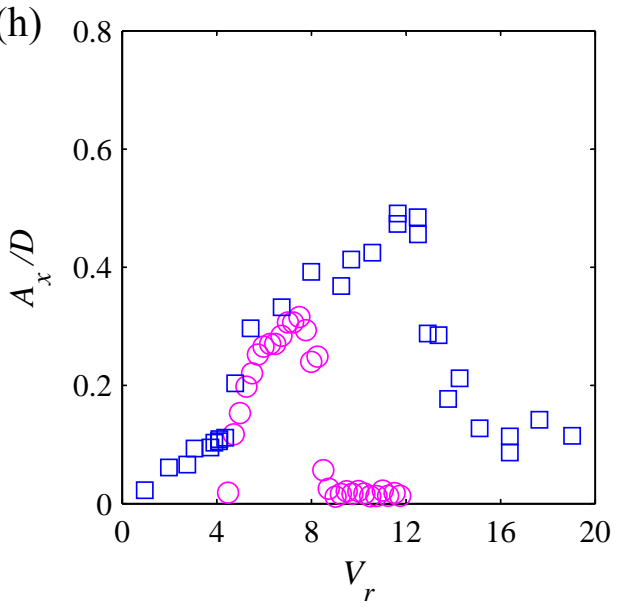

Figure 10 
(a)

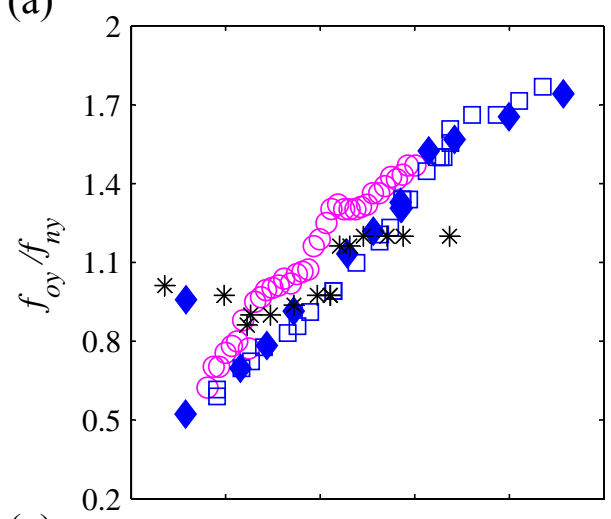

(c)

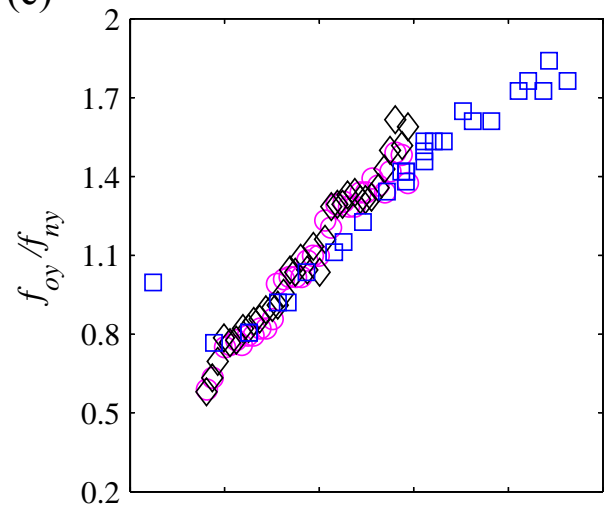

(e)

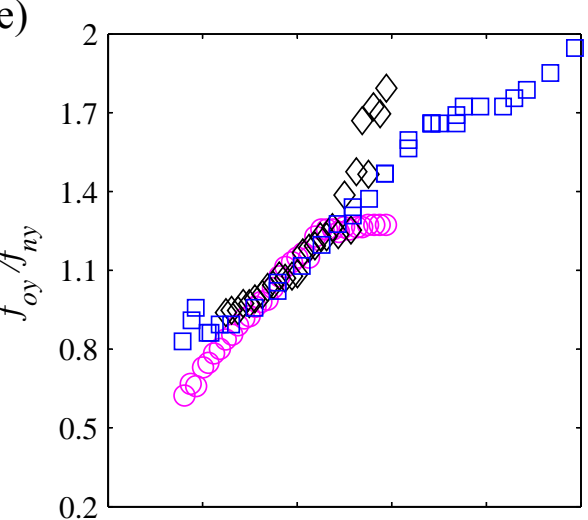

(g)

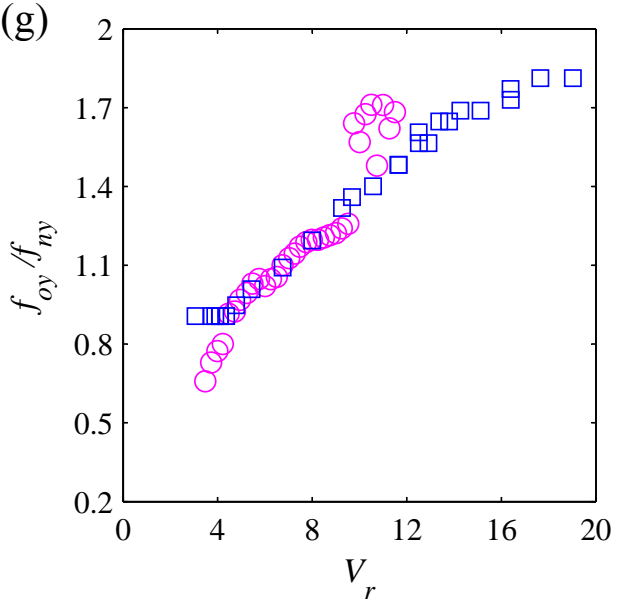

(b)

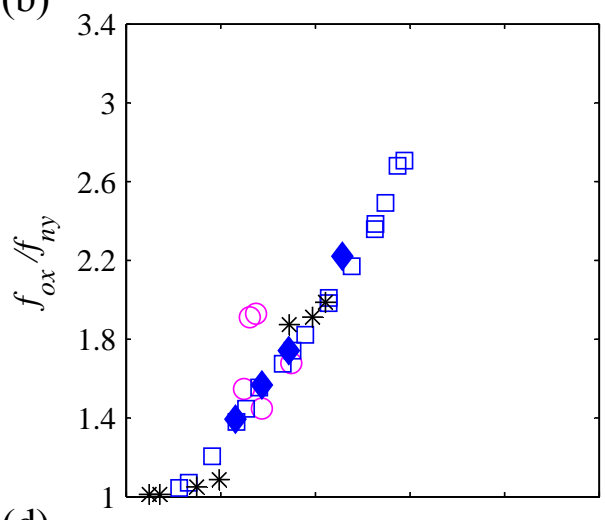

(d)

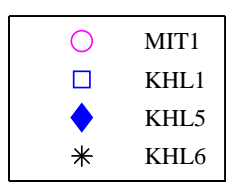

(f)
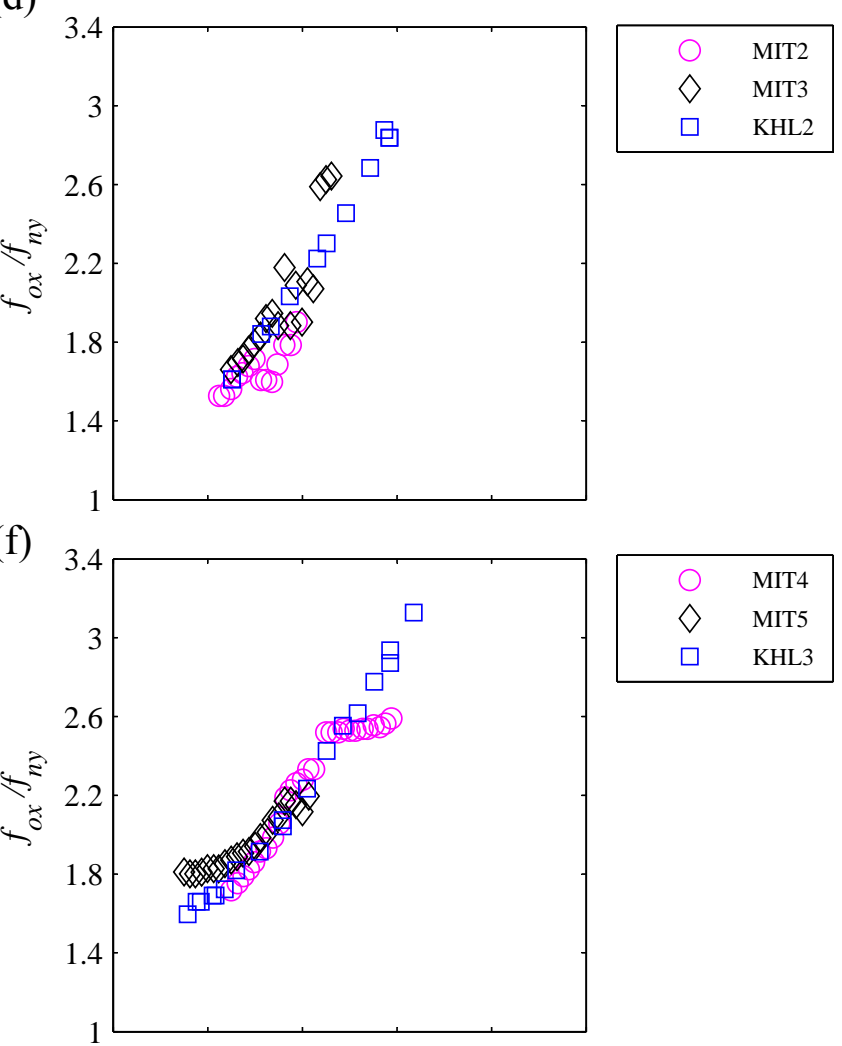

(h)
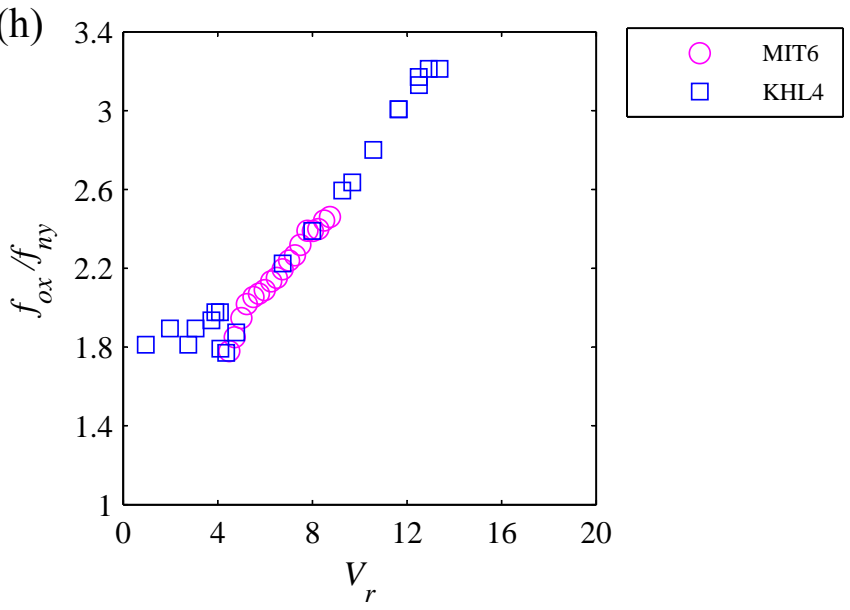

Figure 11 
(a)

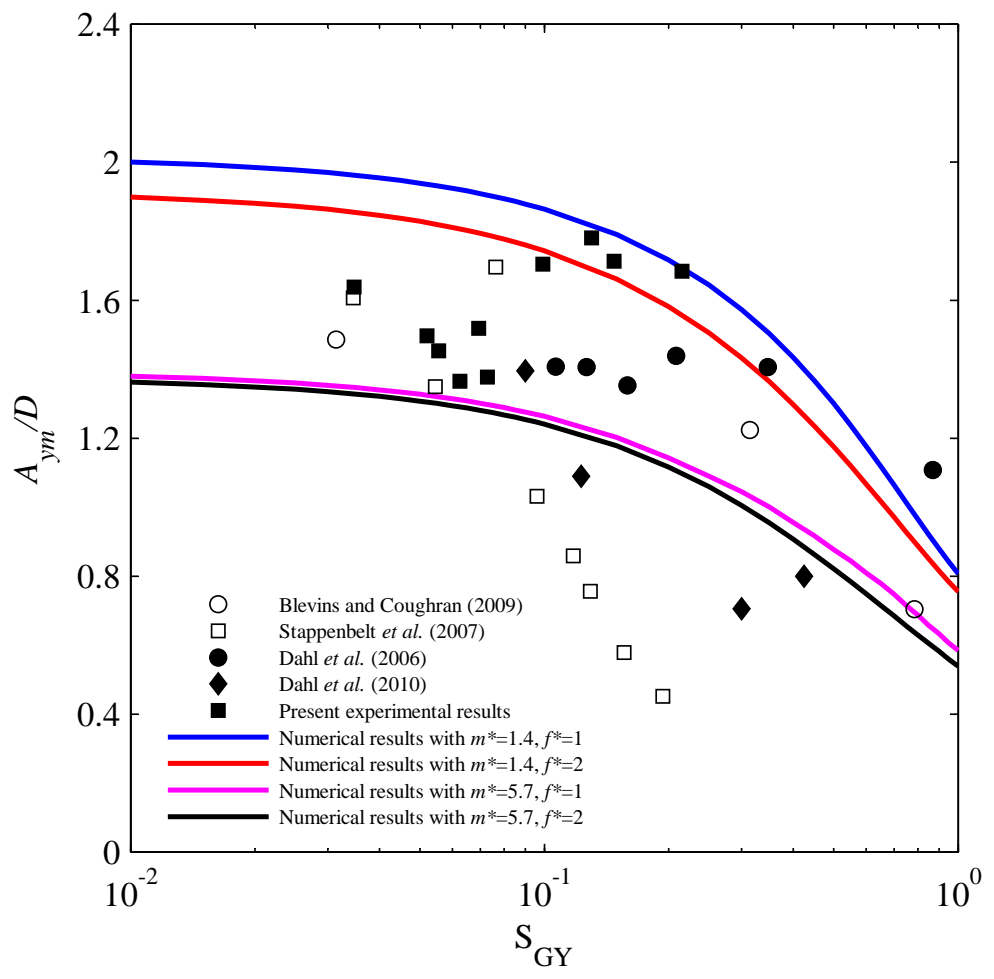

(b)

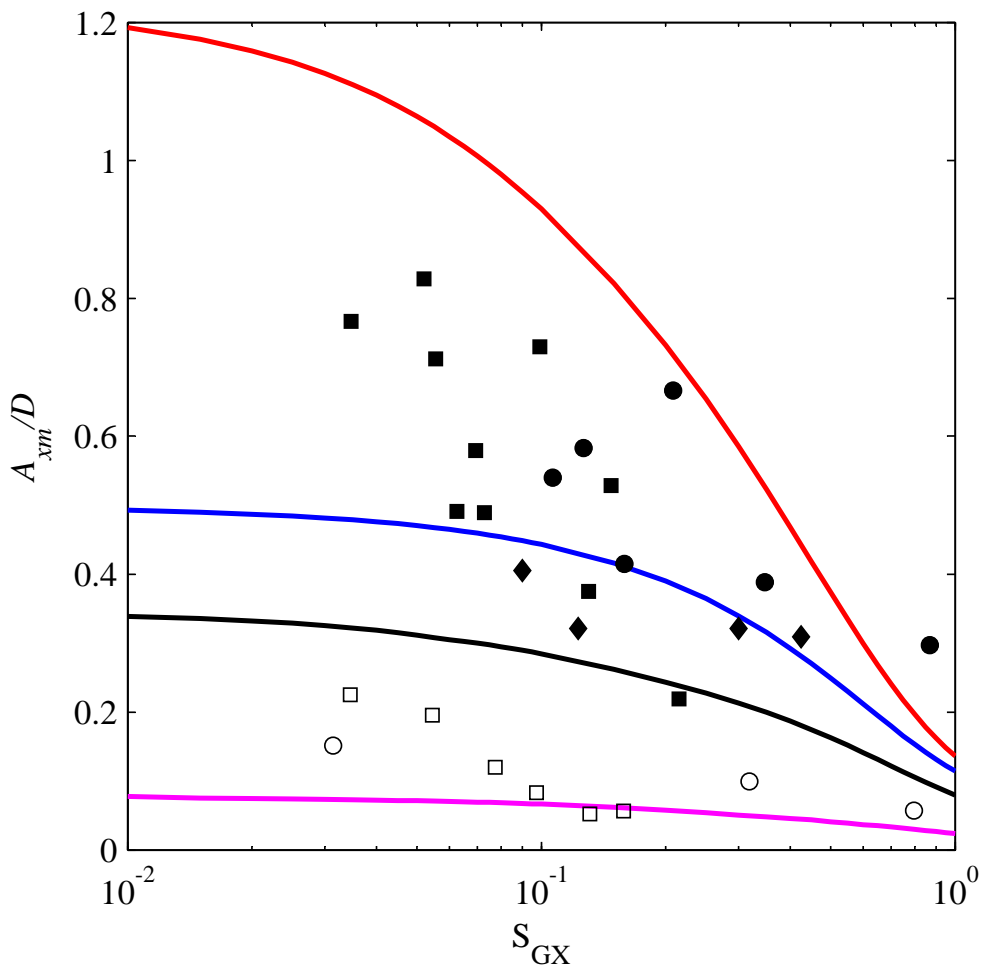

Figure 12 